\title{
ASYMPTOTIC EXPANSIONS OF THE DISTRIBUTION OF THE ESTIMATOR FOR THE GENERALIZED PARTIAL CORRELATION UNDER NONNORMALITY
}

\author{
Haruhiko Ogasawara*
}

\begin{abstract}
The generalized partial correlation is defined as a correlation between two variables, where the linear effects of common and unique third variables are partialed out from the two variables. The generalized partial correlation includes simple, partial, part/semipartial and bipartial correlations as special cases. The Edgeworth expansion of the distribution of the standardized sample coefficient for the generalized partial correlation is obtained up to order $O(1 / n)$ under nonnormality. Also asymptotic expansions of the distribution of the Studentized estimator are obtained using the Edgeworth expansion, Cornish-Fisher expansion and Hall's method with variable transformation. As extensions, the results of multivariate cases or generalized partial set-correlations are given.
\end{abstract}

\section{Introduction}

The partial correlation coefficient and its variations are used to evaluate the strength of association between two variables after the linear effect of a set of the third variable(s) is partialed out. The simple correlation can be seen as a special case when the third variables are null. When the third variables are partialed out only from one of the two variables, the correlation is called the part or semipartial correlation. When different sets of variables are partialed out from the two variables, the correlation becomes the bipartial correlation (Ezekiel, 1941; see also Timm \& Carlson, 1976, p.159).

The problem of the distribution of the sample coefficient of simple correlation has a long history. Under the assumption of the normal distribution, Pearson and Filon (1898, Equation (xl.)) derived the asymptotic covariance of two sample correlations, which has been rediscovered by Girshick (1939, Equation (3.23)), Hsu (1949, p.400), and Olkin and Siotani (1976, Equation (3.1)). Under the same assumption, Fisher (1915) gave the exact distribution of the sample correlation. Under nonnormality, Isserlis (1916, Equation (21)), Hsu (1949, Equation (79)), and Steiger and Hakstian (1982, Equation (3.4)) derived the asymptotic covariance of two sample correlations (see also, Steiger \& Hakstian, 1983). Matrix expressions of the asymptotic covariance matrix of the vectorized sample correlation matrix have been given by Nel (1985, p.143) under normality, Browne and Shapiro (1986, Section 3), Neudecker and Wesselman (1990, Theorem 2) under nonnormality (see also, Kollo \& von Rosen, 2005, Subsection 3.1.4), and Neudecker (1996, Theorem 3) under the elliptical distribution.

Key Words and Phrases: partial correlation, semipartial, bipartial, Edgeworth expansion, Studentized estimators, nonnormality.

* Department of Information and Management Science, Otaru University of Commerce, 3-5-21, Midori, Otaru 047-8501 Japan. Email: hogasa@res.otaru-uc.ac.jp This work was partially supported by Grant-in-Aid for Scientific Research from the Japanese Ministry of Education, Culture, Sports, Science and Technology. 
For the asymptotic expansion of the distribution of the sample correlation, Hotelling (1953, p.212) gave the asymptotic moments up to the sixth order under normality. Cook (1951) derived the moments up to the fourth order under nonnormality. Konishi (1978, Lemma; 1979a, Theorems $2.1 \& 2.2$; 1979b, Theorem 6.2) obtained the Edgeworth expansions of the distributions of the functions of the sample correlation matrix up to order $O\left(n^{-1}\right)$ under normality, where $n+1=N$ is the sample size. Under the same assumption, Niki and Konishi (1984) gave the higher-order Edgeworth expansions of the distribution of the sample correlation while Akahira and Torigie (1998) obtained the corresponding Cornish-Fisher expansion up to order $O\left(n^{-1}\right)$. Under nonnormality Boik (1998, Equation (19)) provided the asymptotic bias of the sample correlation using matrix expression while Ogasawara (2004, Equation (A11)) gave the corresponding elementwise expression. Kollo and Ruul (2003, Theorem 4) derived the multivariate Edgeworth expansion of the vectorized sample correlation matrix up to order $O\left(n^{-1 / 2}\right)$ using the matrix expression of partial derivatives. Nakagawa and Niki (1992), and Ogasawara (2006a) derived the Edgeworth expansion of the sample correlation with different expressions up to order $O\left(n^{-1}\right)$ under nonnormality. Boik and Haaland (2006) gave the similar expansion and that of the Studentized estimator up to order $O\left(n^{-1 / 2}\right)$ using the matrix expression of partial derivatives.

References for the distribution of the sample partial correlation are sparse. It is known that under normality the distribution of the sample partial correlation reduces to that of the sample simple correlation with adjustment for degrees of freedom (Fisher, 1924; see also Anderson, 2003, Theorem 4.3.5; Muirhead, 1982, Theorem 5.3.1). To the author's knowledge, the asymptotic distribution of the sample partial correlation under nonnormality was first given by Steiger and Browne (1984) using the normal approximation with the asymptotic variance for the sample simple correlation between weighted sums of variables under nonnormality. Boik and Haaland (2006) gave the Edgeworth expansion of the sample partial correlation up to order $O\left(n^{-1 / 2}\right)$. For the sample semipartial and bipartial correlations, the asymptotic distributions are not available. However, the test of the hypothesis of zero semipartial correlation is available using the proportional relationship between the semipartial correlation and the corresponding partial correlation (see e.g., van den Burg \& Lewis, 1990, Equation (4)).

The purpose of this article is to give the asymptotic expansions of the distributions of the sample coefficients of various types of partial correlations including the generalized one shown in the next section up to order $O\left(n^{-1}\right)$ and the corresponding expansions for the Studentized estimators up to order $O\left(n^{-1 / 2}\right)$. Simulations will also be carried out to see the usefulness of the asymptotic expansions in finite samples.

\section{Generalized partial correlation}

Lee (1978) extended the bipartial correlation to the case with common and unique variables to be partialed out. Let $\mathbf{x}_{i}(i=1, \ldots, 5)$ be the $p_{i} \times 1$ random vectors with $\operatorname{cov}\left(\mathbf{x}_{i}, \mathbf{x}_{j}^{\prime}\right)=\boldsymbol{\Sigma}_{i j}\left(p_{i} \times p_{j}\right)$ and $\operatorname{cov}\left(\mathbf{x}_{i}\right)=\boldsymbol{\Sigma}_{i i}\left(p_{i} \times p_{i}\right)$. Let $\mathbf{x}_{1 \cdot 34}=\mathbf{x}_{1}-\boldsymbol{\Sigma}_{1,34} \boldsymbol{\Sigma}_{34,34}^{-1}\left(\mathbf{x}_{3}^{\prime}, \mathbf{x}_{4}^{\prime}\right)^{\prime}$ and $\mathbf{x}_{2 \cdot 35}=\mathbf{x}_{2}-\boldsymbol{\Sigma}_{2,35} \boldsymbol{\Sigma}_{35,35}^{-1}\left(\mathbf{x}_{3}^{\prime}, \mathbf{x}_{5}^{\prime}\right)^{\prime}$, where $\boldsymbol{\Sigma}_{1,34}=\operatorname{cov}\left(\mathbf{x}_{1},\left(\mathbf{x}_{3}^{\prime}, \mathbf{x}_{4}^{\prime}\right)\right)\left(p_{1} \times\left(p_{3}+p_{4}\right)\right)$ and 
$\boldsymbol{\Sigma}_{34,34}=\operatorname{cov}\left(\left(\mathbf{x}_{3}^{\prime}, \mathbf{x}_{4}^{\prime}\right)^{\prime}\right)\left(\left(p_{3}+p_{4}\right) \times\left(p_{3}+p_{4}\right)\right)$ with the assumption of positive definite $\boldsymbol{\Sigma}_{34,34}$ and $\boldsymbol{\Sigma}_{35,35}$. That is, the variables in $\mathbf{x}_{3}$ are partialed out from both $\mathbf{x}_{1}$ and $\mathbf{x}_{2}$ while those in $\mathbf{x}_{4}$ and $\mathbf{x}_{5}$ are partialed out only from $\mathbf{x}_{1}$ and $\mathbf{x}_{2}$, respectively.

Lee (1978) defined the $\mathrm{g}_{1^{-}}$and $\mathrm{g}_{2}$-bipartial correlations in the context of canonical correlation analysis for $\mathbf{x}_{1 \cdot 34}$ and $\mathbf{x}_{2 \cdot 35}$. In his definition, the $\mathrm{g}_{1}$-bipartial correlation is for the case with $\mathbf{x}_{3}$ uncorrelated with $\mathbf{x}_{4}$ and $\mathbf{x}_{5}$, while the $\mathrm{g}_{2}$-bipartial correlation is defined when $\mathbf{x}_{3}$ is correlated with $\mathbf{x}_{4}$ and $\mathbf{x}_{5}$. In this article, the generalized partial correlation, denoted by $\rho_{1_{i} \cdot 34,2_{j} \cdot 35}\left(i=1, \ldots, p_{1} ; j=1, \ldots, p_{2}\right)$, is defined irrespective of $\mathbf{x}_{3}$ being uncorrelated or correlated with $\mathbf{x}_{4}$ and $\mathbf{x}_{5}$ :

$$
\rho_{1_{i} \cdot 34,2_{j} \cdot 35}=\left(\boldsymbol{\Sigma}_{1 \cdot 34,2 \cdot 35}\right)_{i j} /\left\{\left(\boldsymbol{\Sigma}_{11 \cdot 34}\right)_{i i}\left(\boldsymbol{\Sigma}_{22 \cdot 35}\right)_{j j}\right\}^{1 / 2} \quad\left(i=1, \ldots, p_{1} ; j=1, \ldots, p_{2}\right),
$$

where $(\cdot)_{i j}$ denotes the $(i, j)$ th element of the argument matrix and

$$
\operatorname{cov}\left(\mathbf{x}_{1 \cdot 34}, \mathbf{x}_{2 \cdot 35}^{\prime}\right)=\boldsymbol{\Sigma}_{1 \cdot 34,2 \cdot 35}, \quad \operatorname{cov}\left(\mathbf{x}_{1 \cdot 34}\right)=\boldsymbol{\Sigma}_{1 \cdot 34,1 \cdot 34} \equiv \boldsymbol{\Sigma}_{11 \cdot 34}, \quad \operatorname{cov}\left(\mathbf{x}_{2 \cdot 35}\right)=\boldsymbol{\Sigma}_{22 \cdot 35} .
$$

The actual expressions of the partial covariance matrices of (2.2) are

$$
\begin{aligned}
& \boldsymbol{\Sigma}_{1 \cdot 34,2 \cdot 35}=\boldsymbol{\Sigma}_{12}-\boldsymbol{\Sigma}_{1,34} \boldsymbol{\Sigma}_{34,34}^{-1} \boldsymbol{\Sigma}_{34,2}-\boldsymbol{\Sigma}_{1,35} \boldsymbol{\Sigma}_{35,35}^{-1} \boldsymbol{\Sigma}_{35,2}+\boldsymbol{\Sigma}_{1,34} \boldsymbol{\Sigma}_{34,34}^{-1} \boldsymbol{\Sigma}_{34,35} \boldsymbol{\Sigma}_{35,35}^{-1} \boldsymbol{\Sigma}_{35,2}, \\
& \boldsymbol{\Sigma}_{11 \cdot 34}=\boldsymbol{\Sigma}_{11}-\boldsymbol{\Sigma}_{1,34} \boldsymbol{\Sigma}_{34,34}^{-1} \boldsymbol{\Sigma}_{34,1}, \quad \boldsymbol{\Sigma}_{22 \cdot 35}=\boldsymbol{\Sigma}_{22}-\boldsymbol{\Sigma}_{2,35} \boldsymbol{\Sigma}_{35,35}^{-1} \boldsymbol{\Sigma}_{35,2} .
\end{aligned}
$$

It is easy to find that the simple, partial, semipartial and bipartial correlations are special cases of the generalized partial correlation of (2.1) when some or all of $\mathbf{x}_{3}, \mathbf{x}_{4}$ and $\mathbf{x}_{5}$ are null. The estimator of $\rho_{1_{i} \cdot 34,2_{j} \cdot 35}$ is given by replacing $\boldsymbol{\Sigma}_{i j}$ 's in (2.3) by the corresponding $p_{i} \times p_{j}$ unbiased sample covariance matrices $\left(\mathbf{S}_{i j}\right.$ 's) with the sample counterparts of $(2.1)$ and (2.2).

\section{Asymptotic expansion of the distribution of the sample generalized partial correlation}

For simplicity of notation, rewrite (2.1) by the generic expression:

$$
\theta=\psi_{3} / \sqrt{\psi_{1} \psi_{2}}
$$

That is, $\theta$ denotes a generalized partial correlation while $\psi_{1}, \psi_{2}$ and $\psi_{3}$ are corresponding partial variances and covariance. Recall that other types of (partial) correlations shown earlier are given as special cases of (3.1). Assume that the estimator $\hat{\theta}$ of $\theta$ is expanded about the true $\theta$ by the Taylor series up to the term of the third derivatives with respect to sample variances and covariances of associated observed variables, i.e. $\mathbf{s}=\mathrm{v}(\mathbf{S})$, where $\mathbf{S}$ is the $p \times p$ sample unbiased covariance matrix with $p=2+p_{3}+p_{4}+p_{5}$ based on $N$ observations for the random vector $\left(X_{1_{i}}, X_{2_{j}}, \mathbf{x}_{3}^{\prime}, \mathbf{x}_{4}^{\prime}, \mathbf{x}_{5}^{\prime}\right)^{\prime}, X_{k_{l}}=\left(\mathbf{x}_{k}\right)_{l},(\cdot)_{l}$ is the $l$-th element of the argument vector, and $\mathrm{v}(\cdot)$ is the vectorizing operator taking the nonduplicated elements of a symmetric matrix.

Then, with the assumption of the existence of the finite moments of the observed variables up to a required order, the cumulants of $w=n^{1 / 2}(\hat{\theta}-\theta)$ up to the fourth order are 
given as

$$
\begin{aligned}
& \kappa_{1}(w)=\mathrm{E}(w)=n^{-1 / 2} \alpha_{1}+o\left(n^{-1 / 2}\right), \\
& \kappa_{2}(w)=\mathrm{E}\left[\{w-\mathrm{E}(w)\}^{2}\right]=\alpha_{2}+n^{-1} \Delta \alpha_{2}+o\left(n^{-1}\right), \\
& \kappa_{3}(w)=\mathrm{E}\left[\{w-\mathrm{E}(w)\}^{3}\right]=n^{-1 / 2} \alpha_{3}+o\left(n^{-1 / 2}\right), \\
& \kappa_{4}(w)=\mathrm{E}\left[\{w-\mathrm{E}(w)\}^{4}\right]-3\left\{\kappa_{2}(w)\right\}^{2}=n^{-1} \alpha_{4}+o\left(n^{-1}\right),
\end{aligned}
$$

where $n^{-1 / 2} \alpha_{1}, \alpha_{2}, n^{-1 / 2} \alpha_{3}$ and $n^{-1} \alpha_{4}$ are the asymptotic cumulants of $w$ up to the fourth order, $n^{-1} \Delta \alpha_{2}$ is the added higher-order asymptotic variance of $w$ (see Fujikoshi, 1980; Ogasawara, 2006a). It is known that $\alpha_{1}, \ldots, \alpha_{4}$ and $\Delta \alpha_{2}$ are given as functions of two sets of arguments. The first set is given by the partial derivatives of $\hat{\theta}$ with respect to s up to the third order evaluated at $\boldsymbol{\sigma}=\mathrm{v}(\boldsymbol{\Sigma})$, where $\boldsymbol{\Sigma}$ is the population counterpart of S. The second set corresponds to the cumulants or moments of the associated observed variables up to the eighth-order. That is, e.g.,

$$
\alpha_{1}=\frac{1}{2} \operatorname{tr}\left(\frac{\partial^{2} \theta}{\partial \boldsymbol{\sigma} \partial \boldsymbol{\sigma}^{\prime}} \boldsymbol{\Omega}\right), \quad \alpha_{2}=\frac{\partial \theta}{\partial \boldsymbol{\sigma}^{\prime}} \boldsymbol{\Omega} \frac{\partial \theta}{\partial \boldsymbol{\sigma}},
$$

where $\boldsymbol{\Omega}=n \operatorname{acov}(\mathbf{s})$ is the $p^{*} \times p^{*}$ matrix with $p^{*}=p(p+1) / 2 ; \operatorname{acov}(\cdot)$ denotes the asymptotic covariance matrix of order $O\left(n^{-1}\right)$ for the argument vector of estimators,

$$
(\boldsymbol{\Omega})_{a b, c d}=\sigma_{a b c d}-\sigma_{a b} \sigma_{c d} \quad(p \geq a \geq b \geq 1 ; p \geq c \geq d \geq 1),
$$

where the double subscript notation for the $(a, b)$ th row and $(c, d)$ th column is used; $\sigma_{a b c d}$ is the fourth multivariate central moment of the variables $X_{a}, X_{b}, X_{c}$ and $X_{d} ; \sigma_{a b}=(\boldsymbol{\Sigma})_{a b}$; and $\partial \theta / \partial \boldsymbol{\sigma}=\partial \hat{\theta} /\left.\partial \mathbf{s}\right|_{\mathbf{s}=\boldsymbol{\sigma}}$ with other similar expressions for simplicity of notation. The remaining expressions for $\alpha_{3}, \alpha_{4}$ and $\Delta \alpha_{2}$ are available (e.g., Ogasawara, 2006a, Section 3; 2006b, Equation (3.4)) but are not repeated here since they are involved.

When the moments or cumulants of the $p$ observed variables up to the eighth order are available, the remaining task to obtain $\alpha_{1}$ to $\alpha_{4}$ and $\Delta \alpha_{2}$ in (3.2) is to derive the partial derivatives of $\hat{\theta}$ with respect to $\mathbf{s}$ up to the third order, which is given in two steps. In the first step, the partial derivatives of $\hat{\theta}$ with respect to $\hat{\psi}_{i}$ 's are derived. Then, in the second step, the partial derivatives of $\hat{\psi}_{i}$ 's with respect to $\mathbf{s}$ are obtained. The results will be given in the appendix.

Approximations to the distribution function of $w$ by the Edgeworth expansion are given by

$$
\begin{aligned}
\operatorname{Pr}\left(\frac{w}{\alpha_{2}^{1 / 2}} \leq z\right)= & \Phi(z)-n^{-1 / 2}\left\{\frac{\alpha_{1}}{\alpha_{2}^{1 / 2}}+\frac{\alpha_{3}}{6 \alpha_{2}^{3 / 2}}\left(z^{2}-1\right)\right\} \phi(z)-n^{-1}\left\{\frac{1}{2}\left(\Delta \alpha_{2}+\alpha_{1}^{2}\right) \frac{z}{\alpha_{2}}\right. \\
& \left.+\left(\frac{\alpha_{4}}{24}+\frac{\alpha_{1} \alpha_{3}}{6}\right) \frac{z^{3}-3 z}{\alpha_{2}^{2}}+\frac{\alpha_{3}^{2}\left(z^{5}-10 z^{3}+15 z\right)}{72 \alpha_{2}^{3}}\right\} \phi(z)+o\left(n^{-1}\right)
\end{aligned}
$$

(Ogasawara, 2006a), where $\phi(z)=(1 / \sqrt{2 \pi}) \exp \left(-z^{2} / 2\right)$ and $\Phi(z)=\int_{-\infty}^{z} \phi(t) d t$. The first term $\Phi(z)$ on the right-hand side of (3.5) gives the usual normal approximation. The 
approximation up to the term of order $O\left(n^{-1 / 2}\right)$ in (3.5) is the single-term Edgeworth expansion. The approximation up to the term of order $O\left(n^{-1}\right)$ in (3.5) is the two-term Edgeworth expansion. It is known that the approximations to the distribution functions by the Edgeworth expansions can be locally decreasing. Hall's (1992) method using variable transformation is free from this phenomenon though the approximation is of the same order as the single-term Edgeworth expansion:

$$
\begin{aligned}
& \operatorname{Pr}\left(\frac{w}{\alpha_{2}^{1 / 2}} \leq z\right)=\Phi\{g(z)\}+o\left(n^{-1 / 2}\right), \\
& g(z)=\frac{2 n^{1 / 2} \alpha_{2}^{3 / 2}}{\alpha_{3}}\left[\left\{\frac{n^{-1 / 2} \alpha_{3}}{6 \alpha_{2}^{3 / 2}}\left(z-\frac{n^{-1 / 2} \alpha_{1}}{\alpha_{2}^{1 / 2}}\right)-1\right\}^{3}-1\right] .
\end{aligned}
$$

In (3.5) and (3.6), the standardized statistic $w / \alpha_{2}^{1 / 2}$ includes the population value $\alpha_{2}$, which is unavailable in practice, while the Studentized or pivotal statistic is available:

$$
t=\frac{n^{1 / 2}(\hat{\theta}-\theta)}{\hat{\alpha}_{2}^{1 / 2}}=\frac{w}{\hat{\alpha}_{2}^{1 / 2}},
$$

whose cumulants up to the third order are

$$
\kappa_{1}(t)=n^{-1 / 2} \alpha_{1}^{\prime}+O\left(n^{-1}\right), \quad \kappa_{2}(t)=1+O\left(n^{-1}\right), \quad \kappa_{3}(t)=n^{-1 / 2} \alpha_{3}^{\prime}+O\left(n^{-3 / 2}\right),
$$

where

$$
\begin{aligned}
& \alpha_{1}^{\prime}=\alpha_{2}^{-1 / 2} \alpha_{1}-\frac{1}{2} \alpha_{2}^{-3 / 2}\left\{\frac{\partial \theta}{\partial \boldsymbol{\sigma}^{\prime}} \boldsymbol{\Omega} \frac{\partial \alpha_{2}}{\partial \boldsymbol{\sigma}}+\frac{\partial \theta}{\partial \boldsymbol{\sigma}^{\prime}} n \operatorname{acov}\left(\mathbf{s}, \mathbf{s}_{(4)}^{\prime}\right) \frac{\partial \alpha_{2}}{\partial \boldsymbol{\sigma}_{(4)}}\right\}, \\
& \alpha_{3}^{\prime}=\alpha_{2}^{-3 / 2} \alpha_{3}-3 \alpha_{2}^{-3 / 2}\left\{\frac{\partial \theta}{\partial \boldsymbol{\sigma}^{\prime}} \boldsymbol{\Omega} \frac{\partial \alpha_{2}}{\partial \boldsymbol{\sigma}}+\frac{\partial \theta}{\partial \boldsymbol{\sigma}^{\prime}} n \operatorname{acov}\left(\mathbf{s}, \mathbf{s}_{(4)}^{\prime}\right) \frac{\partial \alpha_{2}}{\partial \boldsymbol{\sigma}_{(4)}}\right\},
\end{aligned}
$$

where $\mathbf{s}_{(4)}$ is the ${ }_{p+3} C_{4} \times 1$ vector of the nonduplicated sample multivariate fourth moments, $\boldsymbol{\sigma}_{(4)}$ is its population counterpart, and $\operatorname{acov}\left(\mathbf{s}, \mathbf{s}_{(4)}^{\prime}\right)$ is the $p^{*} \times{ }_{p+3} C_{4}$ asymptotic covariance matrix of order $O\left(n^{-1}\right)$ (Ogasawara, 2007).

Let $z_{\tilde{\alpha}}=\Phi^{-1}(1-\tilde{\alpha})$ (e.g., $\left.\tilde{\alpha}=.05\right)$. Then, the confidence interval with the asymptotic confidence coefficient $1-\tilde{\alpha}$ accurate up to order $O\left(n^{-1 / 2}\right)$ by the Cornish-Fisher expansion is

$$
\hat{\theta}+\left[ \pm z_{\tilde{\alpha} / 2}-n^{-1 / 2}\left\{\hat{\alpha}_{1}^{\prime}+\left(\hat{\alpha}_{3}^{\prime} / 6\right)\left(z_{\tilde{\alpha} / 2}^{2}-1\right)\right\}\right] n^{-1 / 2} \hat{\alpha}_{2}^{1 / 2} .
$$

The adjusted Cornish-Fisher expansion gives

$$
\hat{\theta} \pm z_{\tilde{\alpha} / 2} \exp \left[\mp n^{-1 / 2}\left\{\hat{\alpha}_{1}^{\prime}+\left(\hat{\alpha}_{3}^{\prime} / 6\right)\left(z_{\tilde{\alpha} / 2}^{2}-1\right)\right\} / z_{\tilde{\alpha} / 2}\right] n^{-1 / 2} \hat{\alpha}_{2}^{1 / 2}
$$

with the corresponding double signs, where we find that the terms $\pm z_{\tilde{\alpha} / 2} \exp [\cdot] n^{-1 / 2} \hat{\alpha}_{2}^{1 / 2}$ have the same signs as those by the normal approximation (the author is indebted to an anonymous reviewer for this adjustment). The confidence interval by Hall's (1992) method corresponding to $(3.10)$ is

$$
\hat{\theta}-n^{-1} \hat{\alpha}_{2}^{1 / 2} \hat{\alpha}_{1}^{\prime}+6 \hat{\alpha}_{2}^{1 / 2}\left(\hat{\alpha}_{3}^{\prime}\right)^{-1}\left[\left\{1-(1 / 2) \hat{\alpha}_{3}^{\prime}\left( \pm n^{-1 / 2} z_{\tilde{\alpha} / 2}-\left(n^{-1} / 6\right) \hat{\alpha}_{3}^{\prime}\right)\right\}^{1 / 3}-1\right] .
$$


Under normality, (3.9) becomes somewhat simplified:

$$
\begin{aligned}
& \alpha_{\mathrm{NT} 1}^{\prime}=\alpha_{\mathrm{NT} 2}^{-1 / 2} \alpha_{\mathrm{NT} 1}-\frac{1}{2} \alpha_{\mathrm{NT} 2}^{-3 / 2} \frac{\partial \theta}{\partial \boldsymbol{\sigma}^{\prime}} \boldsymbol{\Omega}_{\mathrm{NT}} \frac{\partial \alpha_{\mathrm{NT} 2}}{\partial \boldsymbol{\sigma}}, \\
& \alpha_{\mathrm{NT} 3}^{\prime}=\alpha_{\mathrm{NT} 2}^{-3 / 2} \alpha_{\mathrm{NT} 3}-3 \alpha_{\mathrm{NT} 2}^{-3 / 2} \frac{\partial \theta}{\partial \boldsymbol{\sigma}^{\prime}} \boldsymbol{\Omega}_{\mathrm{NT}} \frac{\partial \alpha_{\mathrm{NT} 2}}{\partial \boldsymbol{\sigma}},
\end{aligned}
$$

where the subscript NT denotes that the subscripted value is given under normality, and $\left(\boldsymbol{\Omega}_{\mathrm{NT}}\right)_{a b, c d}=\sigma_{a c} \sigma_{b d}+\sigma_{a d} \sigma_{b c}$. In practice, the normal-theory Studentized statistic $w / \hat{\alpha}_{\mathrm{NT} 2}^{1 / 2}$ tends to be used inappropriately under nonnormality. In such a case, it is known that (3.9) becomes

$$
\begin{aligned}
& \alpha_{\mathrm{NT} 1}^{\prime \prime}=\alpha_{\mathrm{NT} 2}^{-1 / 2} \alpha_{1}-\frac{1}{2} \alpha_{\mathrm{NT} 2}^{-3 / 2} \frac{\partial \theta}{\partial \boldsymbol{\sigma}^{\prime}} \boldsymbol{\Omega} \frac{\partial \alpha_{\mathrm{NT} 2}}{\partial \boldsymbol{\sigma}}, \quad \alpha_{\mathrm{NT} 2}^{\prime \prime}=\alpha_{\mathrm{NT} 2}^{-1} \alpha_{2}, \\
& \alpha_{\mathrm{NT} 3}^{\prime \prime}=\alpha_{\mathrm{NT} 2}^{-3 / 2} \alpha_{3}-3 \alpha_{\mathrm{NT} 2}^{-5 / 2} \alpha_{2} \frac{\partial \theta}{\partial \boldsymbol{\sigma}^{\prime}} \boldsymbol{\Omega} \frac{\partial \alpha_{\mathrm{NT} 2}}{\partial \boldsymbol{\sigma}}
\end{aligned}
$$

(Ogasawara, 2007).

\section{A numerical example}

An artificial data set using the following $5 \times 5$ population covariance matrix with unit variances is used for illustration:

$$
\boldsymbol{\Sigma}=\left[\begin{array}{rrrrr}
1.00 & \multicolumn{5}{c}{} \\
.64 & 1.00 & \multicolumn{2}{c}{\text { symmetric }} \\
.48 & .48 & 1.00 & & \\
.24 & .24 & .24 & 1.00 & \\
.24 & .24 & .24 & .16 & 1.00
\end{array}\right]
$$

where each $\mathbf{x}_{i}(i=1, \ldots, 5)$ consists of a single variable. The five types of correlation coefficients, $\rho_{12}$ (simple), $\rho_{12 \cdot 3}$ (partial), $\rho_{1 \cdot 34,2 \cdot 3}$ (semipartial), $\rho_{1 \cdot 4,2 \cdot 5}$ (bipartial) and $\rho_{1 \cdot 34,2 \cdot 35}$ (generalized), are considered, where $\rho_{1 \cdot 34,2 \cdot 3}$ is somewhat different from the usual semipartial correlation since $\rho_{1 \cdot 34,2 \cdot 3}$ is a semipartial correlation between $X_{1 \cdot 3}$ and $X_{2 \cdot 3}$ with $X_{4}$ being partialed out only from $X_{1 \cdot 3}$.

Simulations were carried out under normality and nonnormality with the sample sizes $N=50,200$ and 400. Nonnormal observations were generated by $\mathbf{x}=\boldsymbol{\Sigma}^{1 / 2} \mathbf{f}$, where $\boldsymbol{\Sigma}^{1 / 2}$ is a Cholesky-decomposed lower-triangular matrix with $\boldsymbol{\Sigma}=\boldsymbol{\Sigma}^{1 / 2} \boldsymbol{\Sigma}^{1 / 2 \prime}$, and each element of $\mathbf{f}$ is independently chi-square distributed with 1 degree of freedom with standardization to have unit variance. From the generated observations, the five sample correlation coefficients were estimated. This was repeated 1,000,000 times. From the 1,000,000 estimates for each correlation coefficient, the $k$-statistics (unbiased estimators of cumulants) were obtained with multiplication of appropriate powers of $n$ for ease of comparison to the asymptotic values independent of $n$.

Table 1 shows the population coefficients, and the asymptotic and simulated cumulants of their estimators. The simulated $\alpha_{2}^{1 / 2}, \alpha_{1}$ and $\alpha_{3}$ are reasonably similar to their corresponding asymptotic values even when $N$ is as small as 50 while the absolute values 
of simulated $\alpha_{4}$ under nonnormality are smaller than the corresponding asymptotic values though the tendency of the simulated values approaching the asymptotic values with the increase of $N$ is observed. It is seen that the nonnormal cases give larger absolute cumulants than the normal ones, which may be partially explained by the large positive kurtosis of the chi-square distribution with 1 degree of freedom.

Table 2 gives the results of the standard error ratios. In HASE/ASE, HASE = $\left\{\left(\alpha_{2} / n\right)+\left(\Delta \alpha_{2} / n^{2}\right)\right\}^{1 / 2}$ is the higher-order asymptotic standard error, and ASE $=$ $\left(\alpha_{2} / n\right)^{1 / 2}$ is the usual asymptotic standard error while in SD/ASE, SD is the true or simulated standard error given by the square root of the unbiased sample variance based on 1,000,000 estimates for each correlation coefficient. From the table, it is found that under normality, HASE gives values closer to the true ones than ASE while under nonnormality HASE gives somewhat extreme values when $N=50$.

Table 3 shows the results of the normal-theory Studentized estimators under normality and nonnormality. The simulated values with relatively large sample sizes are close to their asymptotic values. It is of interest to find that the signs of the first and third cumulants in Table 3 are positive while they are negative in Table 1.

Table 4 gives the similar results based on Studentized estimates using the asymptotically distribution-free (ADF) theory (see (3.7) with (3.9)) under nonnormality. For the sample asymptotic standard errors, the sample fourth moments of observable variables were used as well as the usual unbiased sample variances and covariances. The number of replications in the simulations was reduced to 100,000 to save computation time. Table 4 shows that when $N=50$, the simulated standard deviations of the Studentized estimates are substantially larger than the unit asymptotic values (compare the corresponding results in Table 3 ).

Table 5 illustrates the accuracy of confidence intervals using sample cumulants in the case of $\rho_{1 \cdot 34,2.35}$ under normality and under nonnormality. The first half of the table is based on the Studentized estimates using normal theory under normality while the second half of the table is given by the Studentized estimates based on the ADF theory under nonnormality. The proportions of the true values below the lower endpoints of confidence intervals are shown with the number of replications being 100,000. In Table 5, three methods are compared: $\mathrm{N}^{*}$ (the usual normal approximation), A.C-F (the adjusted Cornish-Fisher expansion) and Hall (Hall's method using variable transformation). The confidence interval by A.C-F when $z_{.5}=0$ in Table 5 is defined by $\hat{\theta} \pm 0$ (see (3.11)). It is seen that A.C-F and Hall improve over $\mathrm{N}^{*}$ though $\mathrm{N}^{*}$ shows reasonable results in the endpoints with nominal values .9 to .995 under normality. Among the two methods, Hall seems to give results slightly closer to the corresponding nominal values than A.C-F in these data.

Table 6 gives $10^{5} \times$ root mean square errors with respect to the distribution functions of the standardized estimators approximated by $\mathrm{N}^{*}, \mathrm{E} 1$ (the single-term Edgeworth expansion), E2 (the two-term Edgeworth expansion) and Hall's method, where the true values are given by the simulations used in Table 1 and the asymptotic values are given using the population asymptotic cumulants (see (3.4) and (3.5)). Errors are defined as the approximated values minus the corresponding true values at the 40 standardized points, 
Table 1: Simulated and theoretical cumulants of the non-Studentized estimators

\begin{tabular}{lcccccccrr}
\hline Correlation & \multicolumn{2}{c}{$\alpha_{2}^{1 / 2}$ (dispersion) } & \multicolumn{2}{c}{$\alpha_{1}$ (bias) } & \multicolumn{2}{c}{$\alpha_{3}$ (skewness) } & \multicolumn{2}{c}{$\alpha_{4}$ (kurtosis) } \\
coefficient & $N$ & Normal & C1 & Normal & C1 & Normal & C1 & Normal & C1 \\
\hline Simple & 50 & .60 & 1.00 & -.20 & -.39 & -.85 & -3.38 & 3.1 & -1.7 \\
$\rho_{12}$ & 200 & .59 & 1.06 & -.20 & -.43 & -.81 & -4.54 & 2.9 & -.9 \\
$=.640$ & 400 & .59 & 1.08 & -.18 & -.45 & -.79 & -4.86 & 3.2 & -1.2 \\
& Th. & .59 & 1.10 & -.19 & -.45 & -.79 & -5.26 & 2.9 & 12.2 \\
\hline Partial & 50 & .74 & 1.21 & -.20 & -.94 & -1.27 & -5.88 & 4.0 & 10.3 \\
$\rho_{12 \cdot 3}$ & 200 & .72 & 1.25 & -.20 & -1.05 & -1.17 & -7.18 & 3.7 & 17.8 \\
$=.532$ & 400 & .72 & 1.26 & -.20 & -1.08 & -1.16 & -7.49 & 3.5 & 21.7 \\
& Th. & .72 & 1.28 & -.19 & -1.10 & -1.18 & -8.37 & 3.8 & 46.9 \\
\hline Semipartial & 50 & .75 & 1.20 & -.46 & -1.30 & -1.24 & -5.48 & 3.5 & 7.5 \\
$\rho_{1.34,2 \cdot 3}$ & 200 & .73 & 1.24 & -.43 & -1.41 & -1.16 & -7.06 & 3.4 & 15.7 \\
$=.516$ & 400 & .73 & 1.26 & -.43 & -1.42 & -1.18 & -7.56 & 2.9 & 22.0 \\
& Th. & .73 & 1.27 & -.44 & -1.45 & -1.16 & -8.29 & 3.4 & 46.6 \\
\hline Bipartial & 50 & .68 & 1.02 & -.70 & -1.45 & -.94 & -3.17 & 2.4 & -1.0 \\
$\rho_{1.4,2 \cdot 5}$ & 200 & .67 & 1.06 & -.69 & -1.59 & -.89 & -4.56 & 2.2 & 5.0 \\
$=.567$ & 400 & .67 & 1.08 & -.68 & -1.61 & -.90 & -4.95 & 1.9 & 8.5 \\
& Th. & .66 & 1.09 & -.69 & -1.63 & -.90 & -5.53 & 2.5 & 21.0 \\
\hline Generalized & 50 & .76 & 1.20 & -.68 & -1.57 & -1.25 & -5.13 & 3.4 & 3.8 \\
$\rho_{1.34,2 \cdot 35}$ & 200 & .74 & 1.24 & -.65 & -1.66 & -1.17 & -6.77 & 3.3 & 13.0 \\
$=.502$ & 400 & .74 & 1.25 & -.66 & -1.70 & -1.15 & -7.36 & 3.4 & 20.7 \\
& Th. & .74 & 1.27 & -.66 & -1.76 & -1.16 & -8.20 & 3.2 & 47.0 \\
\hline
\end{tabular}

Note. $N=$ The sample size in the simulation, Th. $=$ Theoretical or asymptotic values,

Normal $=$ Normally distributed data, $\mathrm{C} 1=$ Chi-square distributed data with 1 degree of freedom.

$-3.8,-3.6, \ldots, 4.0$. The mean square was taken over the 40 points. In the table, E1, E2 and Hall have errors smaller than $\mathrm{N}^{*}$ and, as a whole, E2 has errors smaller than E1 and Hall while in some cases E2 gives errors larger than E1 and Hall.

\section{Multivariate cases}

The generalized partial correlation (covariance) was initially introduced by Lee (1978) in multivariate form as in (2.3). In this article, the overall or set-correlation indexes between $\mathbf{x}_{1.34}$ and $\mathbf{x}_{2 \cdot 35}$ are considered (for set correlation see Cramer \& Nicewander, 1979; Cohen, 1982; van den burg \& Lewis, 1988; Cohen, Cohen, West \& Aiken, 2003, Chapter 16). One of the basic overall indexes was given by Hotelling (1936, Equation (1.1)) based on Wilks (1932):

$$
\rho_{\mathrm{M} 1}^{2} \equiv 1-\frac{|\boldsymbol{\Psi}|}{\left|\boldsymbol{\Psi}_{11}\right|\left|\boldsymbol{\Psi}_{22}\right|}
$$

where, in the current case, $\boldsymbol{\Psi}=\left[\begin{array}{ll}\boldsymbol{\Psi}_{11} & \boldsymbol{\Psi}_{12} \\ \boldsymbol{\Psi}_{21} & \boldsymbol{\Psi}_{22}\end{array}\right], \boldsymbol{\Psi}_{11}=\boldsymbol{\Sigma}_{11 \cdot 34}, \boldsymbol{\Psi}_{22}=\boldsymbol{\Sigma}_{22 \cdot 35}, \boldsymbol{\Psi}_{12}=\boldsymbol{\Sigma}_{1 \cdot 34,2 \cdot 35}$ and $\boldsymbol{\Psi}_{21}=\boldsymbol{\Psi}_{12}^{\prime}$ (see (2.3)). Note that (5.1), in form, is equal to Rozeboom's (1965) between-set correlation and gives $1-\prod_{i=1}^{q}\left(1-\lambda_{i}^{2}\right)$, where $\lambda_{i}$ is the $i$-th canonical correlation between $\mathbf{x}_{1.34}$ and $\mathbf{x}_{2.35}$ and $q=\min \left(p_{1}, p_{2}\right)$. 
Table 2: Simulated and theoretical standard error ratios of the non-Studentized estimators

\begin{tabular}{lrcccc}
\hline Correlation & \multicolumn{3}{c}{ Normal } & \multicolumn{2}{c}{ C1 } \\
coefficient & $N$ & SD/ASE & HASE/ASE & SD/ASE & HASE/ASE \\
\hline Simple & 50 & 1.023 & 1.023 & .910 & .816 \\
$\rho_{12}$ & 200 & 1.006 & 1.006 & .967 & .958 \\
& 400 & 1.002 & 1.003 & .981 & .979 \\
\hline Partial & 50 & 1.026 & 1.026 & .947 & .870 \\
$\rho_{12 \cdot 3}$ & 200 & 1.006 & 1.006 & .978 & .970 \\
& 400 & 1.003 & 1.003 & .989 & .985 \\
\hline Semipartial & 50 & 1.031 & 1.030 & .943 & .865 \\
$\rho_{1 \cdot 34,2 \cdot 3}$ & 200 & 1.008 & 1.007 & .977 & .968 \\
& 400 & 1.004 & 1.004 & .987 & .984 \\
\hline Bipartial & 50 & 1.027 & 1.027 & .937 & .856 \\
$\rho_{1 \cdot 4,2 \cdot 5}$ & 200 & 1.006 & 1.007 & .976 & .967 \\
& 400 & 1.004 & 1.003 & .987 & .983 \\
\hline Generalized & 50 & 1.035 & 1.034 & .942 & .865 \\
$\rho_{1 \cdot 34,2 \cdot 35}$ & 200 & 1.009 & 1.008 & .976 & .969 \\
& 400 & 1.004 & 1.004 & .986 & .984 \\
\hline
\end{tabular}

Note. $N=$ The sample size in the simulation and the theoretical ratio (HASE $/ \mathrm{ASE}), \mathrm{SD}=$ The standard deviation from the simulation, HASE $=$ $\left\{\left(\alpha_{2} / n\right)+\left(\Delta \alpha_{2} / n^{2}\right)\right\}^{1 / 2}$ with $n=N-1, \operatorname{ASE}=\left(\alpha_{2} / n\right)^{1 / 2}$, Normal = Normally distributed data, $\mathrm{C} 1=$ Chi-square distributed data with 1 degree of freedom.

Another basic overall index is

$$
\rho_{\mathrm{M} 2}^{2} \equiv c^{-1} \operatorname{tr}\left(\boldsymbol{\Psi}_{11}^{-1} \boldsymbol{\Psi}_{12} \boldsymbol{\Psi}_{22}^{-1} \boldsymbol{\Psi}_{21}\right)
$$

where $c$ is a constant. Cramer and Nicewander (1979, Equation (28)) and Takeuchi, Yanai and Mukherjee (1982, Equation (6.68)) used $c=q$. Hooper (1959, 1962) used $c=p_{i}(i=1,2)$ when $\mathbf{x}_{i}$ is a set of criterion variables and $\mathbf{x}_{3-i}$ is a set of explanatory variables. Equation (5.2) is equal to $c^{-1} \sum_{i=1}^{q} \lambda_{i}^{2}$.

The sample values of (5.1) and (5.2) are given by replacing $\boldsymbol{\Psi}$ with its sample counterpart. The asymptotic expansions of the distributions of $\hat{\rho}_{\mathrm{M} 1}^{2}$ and $\hat{\rho}_{\mathrm{M} 2}^{2}$ are available when the partial derivatives with respect to $s_{a b}$ 's are provided, which will be given in the appendix.

\section{Appendix. The partial derivatives}

The following partial derivatives are those evaluated at the population values.

\section{A. The partial derivatives of $\hat{\theta}$ with respect to $\mathrm{s}$}

A.1 The recursive expressions of the partial derivatives of $\hat{\theta}$ with respect to $s$

$$
\frac{\partial \theta}{\partial \sigma_{a b}}=\sum_{i=1}^{3} \frac{\partial \theta}{\partial \psi_{i}} \frac{\partial \psi_{i}}{\partial \sigma_{a b}}, \quad \frac{\partial^{2} \theta}{\partial \sigma_{a b} \partial \sigma_{c d}}=\sum_{i=1}^{3}\left(\sum_{j=1}^{3} \frac{\partial^{2} \theta}{\partial \psi_{i} \partial \psi_{j}} \frac{\partial \psi_{i}}{\partial \sigma_{a b}} \frac{\partial \psi_{j}}{\partial \sigma_{c d}}+\frac{\partial \theta}{\partial \psi_{i}} \frac{\partial^{2} \psi_{i}}{\partial \sigma_{a b} \partial \sigma_{c d}}\right),
$$


Table 3: Simulated and theoretical cumulants of the normal-theory Studentized estimators

\begin{tabular}{|c|c|c|c|c|c|c|c|}
\hline \multirow{2}{*}{$\begin{array}{l}\text { Correlation } \\
\text { coefficient }\end{array}$} & \multicolumn{2}{|c|}{$\alpha_{\mathrm{NT} 2}^{1 / 2}$} & \multirow{2}{*}{$\begin{array}{c}\alpha_{\mathrm{NT} 2}^{1 / 2} \\
\mathrm{C} 1\end{array}$} & \multirow{2}{*}{$\begin{array}{c}\alpha_{\mathrm{NT} 1}^{\prime} \\
\text { Normal }\end{array}$} & \multirow{2}{*}{$\begin{array}{c}\alpha_{\mathrm{NT} 1}^{\prime \prime} \\
\mathrm{C} 1\end{array}$} & \multirow{2}{*}{$\begin{array}{c}\alpha_{\mathrm{NT} 3}^{\prime} \\
\text { Normal }\end{array}$} & \multirow{2}{*}{$\begin{array}{c}\alpha_{\mathrm{NT} 3}^{\prime \prime} \\
\mathrm{C} 1\end{array}$} \\
\hline & $N$ & Normal & & & & & \\
\hline \multirow{4}{*}{$\begin{array}{l}\text { Simple } \\
\rho_{12}\end{array}$} & 50 & 1.08 & 2.37 & 1.01 & 3.87 & 5.5 & 205.0 \\
\hline & 200 & 1.02 & 1.96 & .97 & 3.67 & 4.2 & 85.1 \\
\hline & 400 & 1.01 & 1.90 & .99 & 3.63 & 4.1 & 74.5 \\
\hline & Th. & 1 & 1.86 & .96 & 3.67 & 3.8 & 66.3 \\
\hline \multirow{4}{*}{$\begin{array}{l}\text { Partial } \\
\rho_{12 \cdot 3}\end{array}$} & 50 & 1.09 & 2.10 & .86 & 2.03 & 4.8 & 98.3 \\
\hline & 200 & 1.02 & 1.84 & .81 & 1.85 & 3.6 & 50.7 \\
\hline & 400 & 1.01 & 1.81 & .80 & 1.84 & 3.4 & 46.8 \\
\hline & Th. & 1 & 1.78 & .80 & 1.85 & 3.2 & 41.5 \\
\hline \multirow{4}{*}{$\begin{array}{l}\text { Semipartial } \\
\rho_{1 \cdot 34,2 \cdot 3}\end{array}$} & 50 & 1.08 & 1.92 & .40 & .96 & 4.0 & 53.5 \\
\hline & 200 & 1.02 & 1.78 & .41 & 1.02 & 3.2 & 39.1 \\
\hline & 400 & 1.01 & 1.77 & .41 & 1.08 & 3.0 & 37.6 \\
\hline & Th. & 1 & 1.75 & .39 & 1.11 & 3.0 & 35.6 \\
\hline \multirow{4}{*}{$\begin{array}{l}\text { Bipartial } \\
\rho_{1 \cdot 4,2 \cdot 5}\end{array}$} & 50 & 1.06 & 1.65 & -.12 & -.12 & 3.2 & 22.2 \\
\hline & 200 & 1.02 & 1.63 & -.08 & .05 & 2.9 & 22.2 \\
\hline & 400 & 1.01 & 1.63 & -.07 & .13 & 2.8 & 23.3 \\
\hline & Th. & 1 & 1.64 & -.08 & .22 & 2.7 & 24.3 \\
\hline \multirow{4}{*}{$\begin{array}{l}\text { Generalized } \\
\rho_{1 \cdot 34,2 \cdot 35}\end{array}$} & 50 & 1.08 & 1.82 & .02 & .23 & 3.5 & 36.9 \\
\hline & 200 & 1.02 & 1.74 & .06 & .44 & 3.0 & 32.5 \\
\hline & 400 & 1.01 & 1.73 & .05 & .46 & 2.9 & 31.5 \\
\hline & Th. & 1 & 1.72 & .04 & .48 & 2.8 & 30.7 \\
\hline
\end{tabular}

Note. $N=$ The sample size in the simulation, Th. = Theoretical or asymptotic values, Normal $=$ Normally distributed data, $\mathrm{C} 1=$ Chisquare distributed data with 1 degree of freedom.

$$
\begin{aligned}
& \frac{\partial^{3} \theta}{\partial \sigma_{a b} \partial \sigma_{c d} \partial \sigma_{e f}}= \sum_{i=1}^{3}\left\{\sum _ { j = 1 } ^ { 3 } \left(\sum_{k=1}^{3} \frac{\partial^{3} \theta}{\partial \psi_{i} \partial \psi_{j} \partial \psi_{k}} \frac{\partial \psi_{i}}{\partial \sigma_{a b}} \frac{\partial \psi_{j}}{\partial \sigma_{c d}} \frac{\partial \psi_{k}}{\partial \sigma_{e f}}\right.\right. \\
&\left.\left.+\sum^{3} \frac{\partial^{2} \theta}{\partial \psi_{i} \partial \psi_{j}} \frac{\partial \psi_{i}}{\partial \sigma_{a b}} \frac{\partial^{2} \psi_{j}}{\partial \sigma_{c d} \partial \sigma_{e f}}\right)+\frac{\partial \theta}{\partial \psi_{i}} \frac{\partial^{3} \psi_{i}}{\partial \sigma_{a b} \partial \sigma_{c d} \partial \sigma_{e f}}\right\} \\
&(p \geq a \geq b \geq 1 ; p \geq c \geq d \geq 1 ; p \geq e \geq f \geq 1),
\end{aligned}
$$

where $\sum^{k}$ denotes the sum of $k$ terms with similar patterns.

A.2 The partial derivatives of $\hat{\theta}$ with respect to $\hat{\psi}_{i}$ 's

$$
\begin{aligned}
& \frac{\partial \theta}{\partial\left(\psi_{1}, \psi_{2}, \psi_{3}\right)}=\left(-\frac{1}{2} \psi_{1}^{-3 / 2} \psi_{2}^{-1 / 2} \psi_{3},-\frac{1}{2} \psi_{1}^{-1 / 2} \psi_{2}^{-3 / 2} \psi_{3},\left(\psi_{1} \psi_{2}\right)^{-1 / 2}\right), \\
& \frac{\partial^{2} \theta}{\partial \psi_{1}^{2}}=\frac{3}{4} \psi_{1}^{-5 / 2} \psi_{2}^{-1 / 2} \psi_{3}, \quad \frac{\partial^{2} \theta}{\partial \psi_{2} \partial \psi_{1}}=\frac{1}{4} \psi_{1}^{-3 / 2} \psi_{2}^{-3 / 2} \psi_{3}, \quad \frac{\partial^{2} \theta}{\partial \psi_{2}^{2}}=\frac{3}{4} \psi_{1}^{-1 / 2} \psi_{2}^{-5 / 2} \psi_{3}, \\
& \frac{\partial^{2} \theta}{\partial \psi_{3} \partial \psi_{1}}=-\frac{1}{2} \psi_{1}^{-3 / 2} \psi_{2}^{-1 / 2}, \quad \frac{\partial^{2} \theta}{\partial \psi_{3} \partial \psi_{2}}=-\frac{1}{2} \psi_{1}^{-1 / 2} \psi_{2}^{-3 / 2}, \quad \frac{\partial^{2} \theta}{\partial \psi_{3}^{2}}=0, \\
& \frac{\partial^{3} \theta}{\partial \psi_{1}^{3}}=-\frac{15}{8} \psi_{1}^{-7 / 2} \psi_{2}^{-1 / 2} \psi_{3}, \quad \frac{\partial^{3} \theta}{\partial \psi_{2} \partial \psi_{1}^{2}}=-\frac{3}{8} \psi_{1}^{-5 / 2} \psi_{2}^{-3 / 2} \psi_{3},
\end{aligned}
$$


Table 4: Simulated and theoretical cumulants of the ADF-theory Studentized estimators

\begin{tabular}{|c|c|c|c|c|}
\hline \multirow{2}{*}{$\begin{array}{l}\text { Correlation } \\
\text { coefficient }\end{array}$} & \multicolumn{4}{|c|}{$\mathrm{C} 1$} \\
\hline & $N$ & $\alpha_{2}^{1 / 2 \prime}$ & $\alpha_{1}^{\prime}$ & $\alpha_{3}^{\prime}$ \\
\hline \multirow{4}{*}{$\begin{array}{l}\text { Simple } \\
\rho_{12}\end{array}$} & 50 & 1.69 & 1.72 & 44.1 \\
\hline & 200 & 1.19 & 1.11 & 8.7 \\
\hline & 400 & 1.10 & .99 & 6.1 \\
\hline & Th. & 1 & .92 & 4.0 \\
\hline \multirow{4}{*}{$\begin{array}{l}\text { Partial } \\
\rho_{12 \cdot 3}\end{array}$} & 50 & 1.62 & 1.16 & 37.9 \\
\hline & 200 & 1.19 & .96 & 11.3 \\
\hline & 400 & 1.10 & 1.00 & 9.2 \\
\hline & Th. & 1 & 1.14 & 7.9 \\
\hline \multirow{4}{*}{$\begin{array}{l}\text { Semipartial } \\
\rho_{1 \cdot 34,2 \cdot 3}\end{array}$} & 50 & 1.59 & .61 & 30.6 \\
\hline & 200 & 1.18 & .55 & 11.6 \\
\hline & 400 & 1.10 & .64 & 8.8 \\
\hline & Th. & 1 & .92 & 8.3 \\
\hline \multirow{4}{*}{$\begin{array}{l}\text { Bipartial } \\
\rho_{1 \cdot 4,2 \cdot 5}\end{array}$} & 50 & 1.49 & -.14 & 18.3 \\
\hline & 200 & 1.17 & .09 & 10.1 \\
\hline & 400 & 1.10 & .21 & 8.1 \\
\hline & Th. & 1 & .48 & 7.6 \\
\hline \multirow{4}{*}{$\begin{array}{l}\text { Generalized } \\
\rho_{1 \cdot 34,2 \cdot 35}\end{array}$} & 50 & 1.57 & .22 & 27.1 \\
\hline & 200 & 1.19 & .40 & 11.1 \\
\hline & 400 & 1.10 & .49 & 9.1 \\
\hline & Th. & 1 & .71 & 8.6 \\
\hline
\end{tabular}

Note. $N=$ The sample size in the simulation, Th. $=$

Theoretical or asymptotic values, $\mathrm{C} 1=$ Chi-square distributed data with 1 degree of freedom.

$$
\begin{aligned}
& \frac{\partial^{3} \theta}{\partial \psi_{2}^{2} \partial \psi_{1}}=-\frac{3}{8} \psi_{1}^{-3 / 2} \psi_{2}^{-5 / 2} \psi_{3}, \\
& \frac{\partial^{3} \theta}{\partial \psi_{2}^{3}}=-\frac{15}{8} \psi_{1}^{-1 / 2} \psi_{2}^{-7 / 2} \psi_{3}, \quad \frac{\partial^{3} \theta}{\partial \psi_{3} \partial \psi_{1}^{2}}=\frac{3}{4} \psi_{1}^{-5 / 2} \psi_{2}^{-1 / 2}, \\
& \frac{\partial^{3} \theta}{\partial \psi_{3} \partial \psi_{2} \partial \psi_{1}}=\frac{1}{4} \psi_{1}^{-3 / 2} \psi_{2}^{-3 / 2}, \\
& \frac{\partial^{3} \theta}{\partial \psi_{3} \partial \psi_{2}^{2}}=\frac{3}{4} \psi_{1}^{-1 / 2} \psi_{2}^{-5 / 2}, \quad \frac{\partial^{3} \theta}{\partial \psi_{3}^{2} \partial \psi_{1}}=\frac{\partial^{3} \theta}{\partial \psi_{3}^{2} \partial \psi_{2}}=\frac{\partial^{3} \theta}{\partial \psi_{3}^{3}}=0 .
\end{aligned}
$$

\section{A.3 The partial derivatives of $\hat{\psi}_{i}$ 's with respect to $s$}

$$
\begin{aligned}
& \text { Let } \boldsymbol{\sigma}_{34,1}=\operatorname{cov}\left(\left(\mathbf{x}_{3}^{\prime}, \mathbf{x}_{4}^{\prime}\right)^{\prime}, X_{1_{i}}\right), \boldsymbol{\sigma}_{35,2}=\operatorname{cov}\left(\left(\mathbf{x}_{3}^{\prime}, \mathbf{x}_{5}^{\prime}\right)^{\prime}, X_{2_{j}}\right), \\
& \sigma_{11}=\operatorname{var}\left(X_{1_{i}}\right), \sigma_{12}=\operatorname{cov}\left(X_{1_{i}}, X_{2_{j}}\right), \sigma_{22}=\operatorname{var}\left(X_{2_{j}}\right)\left(i=1, \ldots, p_{1} ; j=1, \ldots, p_{2}\right), \\
& \begin{aligned}
\psi_{1}=\psi_{1 \mathrm{~A}}-\psi_{1 \mathrm{~B}} \equiv \sigma_{11}-\boldsymbol{\sigma}_{34,1}^{\prime} \boldsymbol{\Sigma}_{34,34}^{-1} \boldsymbol{\sigma}_{34,1}, \psi_{2}=\psi_{2 \mathrm{~A}}-\psi_{2 \mathrm{~B}} \equiv \sigma_{22}-\boldsymbol{\sigma}_{35,2}^{\prime} \boldsymbol{\Sigma}_{35,35}^{-1} \boldsymbol{\sigma}_{35,2}, \\
\quad \psi_{3}=\psi_{3 \mathrm{~A}}-\psi_{3 \mathrm{~B}}-\psi_{3 \mathrm{C}}+\psi_{3 \mathrm{D}} \\
\quad \equiv \sigma_{12}-\boldsymbol{\sigma}_{34,1}^{\prime} \boldsymbol{\Sigma}_{34,34}^{-1} \boldsymbol{\sigma}_{34,2}-\boldsymbol{\sigma}_{35,1}^{\prime} \boldsymbol{\Sigma}_{35,35}^{-1} \boldsymbol{\sigma}_{35,2}+\boldsymbol{\sigma}_{34,1}^{\prime} \boldsymbol{\Sigma}_{34,34}^{-1} \boldsymbol{\Sigma}_{34,35} \boldsymbol{\Sigma}_{35,35}^{-1} \boldsymbol{\sigma}_{35,2},
\end{aligned}
\end{aligned}
$$

where the subscripts $i$ and $j$ are temporarily suppressed in e.g., $\sigma_{12}$ for simplicity of nota- 
Table 5: Simulated proportions below the lower endpoints of the confidence intervals for $\rho_{1 \cdot 34,2 \cdot 35}$

\begin{tabular}{|c|c|c|c|c|c|c|c|c|}
\hline \multirow[b]{2}{*}{$N$} & \multirow[b]{2}{*}{ Method } & \multicolumn{7}{|c|}{ Nominal values } \\
\hline & & .0050 & .0250 & .1000 & .5000 & .9000 & .9750 & .9950 \\
\hline \multicolumn{9}{|c|}{ Based on the normal-theory Studentized estimates under normality } \\
\hline \multirow{3}{*}{50} & $\mathrm{~N}^{*}$ & .0166 & .0441 & .1166 & .4779 & .8950 & .9756 & .9953 \\
\hline & A.C-F & .0071 & .0320 & .1114 & .4779 & .8882 & .9699 & .9936 \\
\hline & Hall & .0037 & .0280 & .1108 & .5001 & .8885 & .9702 & .9937 \\
\hline \multirow{3}{*}{200} & $\mathrm{~N}^{*}$ & .0099 & .0320 & 1052 & .4909 & .9007 & .9786 & .9962 \\
\hline & A.C-F & .0059 & .0263 & . 1014 & .4909 & .8965 & .9742 & .9945 \\
\hline & Hall & .0054 & .0257 & .1013 & .5024 & .8965 & .9744 & .9946 \\
\hline \multirow{3}{*}{400} & $\mathrm{~N}^{*}$ & .0075 & .0298 & .1045 & .4920 & .9019 & .9782 & .9964 \\
\hline & A.C-F & .0050 & .0253 & .1021 & .4920 & .8991 & .9751 & .9950 \\
\hline & Hall & .0048 & .0250 & . 1020 & .5003 & .8991 & .9752 & .9951 \\
\hline
\end{tabular}

Based on the ADF-theory Studentized estimates under nonnormality (chi-square distributed data with 1 degree of freedom)

\begin{tabular}{lllllllll}
\hline \multirow{2}{*}{50} & $\mathrm{~N}^{*}$ & .0605 & .0994 & .1744 & .4590 & .8244 & .9304 & .9736 \\
& A.C-F & .0367 & .0814 & .1739 & .4590 & .8312 & .9338 & .9756 \\
& Hall & .0180 & .0626 & .1722 & .4910 & .8305 & .9350 & .9771 \\
\hline \multirow{2}{*}{200} & $\mathrm{~N}^{*}$ & .0268 & .0595 & .1380 & .4804 & .8808 & .9681 & .9925 \\
& A.C-F & .0176 & .0510 & .1386 & .4804 & .8759 & .9618 & .9896 \\
& Hall & .0146 & .0485 & .1383 & .5030 & .8758 & .9622 & .9900 \\
\hline \multirow{2}{*}{400} & $\mathrm{~N}^{*}$ & .0177 & .0479 & .1262 & .4878 & .8920 & .9734 & .9949 \\
& A.C-F & .0128 & .0417 & .1261 & .4878 & .8852 & .9662 & .9914 \\
& Hall & .0118 & .0407 & .1260 & .5051 & .8849 & .9667 & .9919 \\
\hline
\end{tabular}

Note. $N=$ The sample size in the simulation, $\mathrm{N}^{*}=$ Normal approximation, A.C-F $=$ The adjusted Cornish-Fisher expansion, Hall $=$ Hall's method by variable transformation.

tion. Then, the nonzero partial derivatives of $\hat{\psi}_{1 \mathrm{~A}}$ through $\hat{\psi}_{3 \mathrm{D}}$ with respect to $s_{a b}$ 's are as follows:

$$
\begin{aligned}
\frac{\partial \psi_{1 \mathrm{~A}}}{\partial \sigma_{11}} & =1 \\
\frac{\partial \psi_{1 \mathrm{~B}}}{\partial \sigma_{a b}} & =\frac{\partial \boldsymbol{\sigma}_{34,1}^{\prime}}{\partial \sigma_{a b}} \boldsymbol{\Sigma}_{34,34}^{-1} \boldsymbol{\sigma}_{34,1}+\boldsymbol{\sigma}_{34,1}^{\prime} \frac{\partial \boldsymbol{\Sigma}_{34,34}^{-1}}{\partial \sigma_{a b}} \boldsymbol{\sigma}_{34,1}+\boldsymbol{\sigma}_{34,1}^{\prime} \boldsymbol{\Sigma}_{34,34}^{-1} \frac{\partial \boldsymbol{\sigma}_{34,1}}{\partial \sigma_{a b}} \\
& \equiv \sum \frac{\partial \boldsymbol{\sigma}_{34,1}^{\prime}}{\partial \sigma_{a b}} \boldsymbol{\Sigma}_{34,34}^{-1} \boldsymbol{\sigma}_{34,1}, \\
\frac{\partial \psi_{2 \mathrm{~A}}}{\partial \sigma_{22}} & =1, \quad \frac{\partial \psi_{2 \mathrm{~B}}}{\partial \sigma_{a b}}=\sum^{3} \frac{\partial \boldsymbol{\sigma}_{35,2}^{\prime}}{\partial \sigma_{a b}} \boldsymbol{\Sigma}_{35,35}^{-1} \boldsymbol{\sigma}_{35,2}, \quad \frac{\partial \psi_{3 \mathrm{~A}}}{\partial \sigma_{21}}=1, \\
\frac{\partial \psi_{3 \mathrm{~B}}}{\partial \sigma_{a b}} & =\sum \frac{\partial \boldsymbol{\sigma}_{34,1}^{\prime}}{\partial \sigma_{a b}} \boldsymbol{\Sigma}_{34,34}^{-1} \boldsymbol{\sigma}_{34,2}, \quad \\
\frac{\partial \psi_{3 \mathrm{C}}}{\partial \sigma_{a b}} & =\sum \frac{\partial \boldsymbol{\sigma}_{35,1}^{\prime}}{\partial \sigma_{a b}} \boldsymbol{\Sigma}_{35,35}^{-1} \boldsymbol{\sigma}_{35,2}, \quad \frac{\partial \psi_{3 \mathrm{D}}}{\partial \sigma_{a b}}=\sum^{5} \frac{\partial \boldsymbol{\sigma}_{34,1}^{\prime}}{\partial \sigma_{a b}} \boldsymbol{\Sigma}_{34,34}^{-1} \boldsymbol{\Sigma}_{34,35} \boldsymbol{\Sigma}_{35,35}^{-1} \boldsymbol{\sigma}_{35,2},
\end{aligned}
$$


Table 6 : $10^{5} \times$ Root mean square errors of the asymptotic distribution functions of the standardized estimators

\begin{tabular}{|c|c|c|c|c|c|c|c|c|}
\hline \multirow{2}{*}{$\begin{array}{l}\text { Correlation } \\
\text { coefficient }\end{array}$} & \multicolumn{4}{|c|}{ Normal } & \multicolumn{4}{|c|}{ Chi-square with 1 degree of freedom } \\
\hline & $\mathrm{N}^{*}$ & E1 & $\mathrm{E} 2$ & Hall & $\mathrm{N}^{*}$ & E1 & $\mathrm{E} 2$ & Hall \\
\hline \multicolumn{9}{|l|}{$N=50$} \\
\hline$\rho_{12}$ & 1226 & 170 & 47 & 200 & 1748 & 1238 & 1123 & 1244 \\
\hline$\rho_{12 \cdot 3}$ & 1056 & 228 & 36 & 266 & 1887 & 821 & 1116 & 828 \\
\hline$\rho_{1 \cdot 34,2 \cdot 3}$ & 1410 & 282 & 50 & 333 & 2486 & 858 & 1169 & 858 \\
\hline$\rho_{1 \cdot 4,2 \cdot 5}$ & 2346 & 271 & 34 & 296 & 3277 & 920 & 1231 & 946 \\
\hline$\rho_{1 \cdot 34,2 \cdot 35}$ & 2043 & 361 & 51 & 397 & 3017 & 862 & 1200 & 878 \\
\hline \multicolumn{9}{|l|}{$N=200$} \\
\hline$\rho_{12}$ & 614 & 44 & 20 & 49 & 756 & 428 & 143 & 429 \\
\hline$\rho_{12 \cdot 3}$ & 496 & 55 & 24 & 62 & 962 & 309 & 164 & 302 \\
\hline$\rho_{1 \cdot 34,2 \cdot 3}$ & 663 & 69 & 11 & 82 & 1273 & 326 & 172 & 319 \\
\hline$\rho_{1 \cdot 4,2 \cdot 5}$ & 1143 & 67 & 14 & 74 & 1690 & 337 & 179 & 335 \\
\hline$\rho_{1 \cdot 34,2 \cdot 35}$ & 970 & 94 & 34 & 103 & 1515 & 332 & 175 & 328 \\
\hline \multicolumn{9}{|l|}{$N=400$} \\
\hline$\rho_{12}$ & 419 & 32 & 29 & 27 & 502 & 237 & 50 & 237 \\
\hline$\rho_{12 \cdot 3}$ & 353 & 31 & 13 & 36 & 675 & 168 & 76 & 163 \\
\hline$\rho_{1 \cdot 34,2 \cdot 3}$ & 466 & 44 & 20 & 53 & 882 & 181 & 67 & 176 \\
\hline$\rho_{1 \cdot 4,2 \cdot 5}$ & 803 & 44 & 21 & 52 & 1182 & 181 & 77 & 181 \\
\hline$\rho_{1 \cdot 34,2 \cdot 35}$ & 690 & 45 & 15 & 48 & 1080 & 208 & 48 & 202 \\
\hline
\end{tabular}

Note. $N=$ The sample size in the simulation, $\mathrm{N}^{*}=$ Normal approximation, $\mathrm{E} 1=$ The single-term Edgeworth expansion, E2 = The two-term Edgeworth expansion, Hall = Hall's method by variable transformation.

$$
\begin{aligned}
\frac{\partial^{2} \psi_{1 \mathrm{~B}}}{\partial \sigma_{a b} \partial \sigma_{c d}}= & \sum \frac{\partial \boldsymbol{\sigma}_{34,1}^{\prime}}{\partial \sigma_{a b}} \frac{\partial \boldsymbol{\Sigma}_{34,34}^{-1}}{\partial \sigma_{c d}} \boldsymbol{\sigma}_{34,1}+\boldsymbol{\sigma}_{34,1}^{\prime} \frac{\partial^{2} \boldsymbol{\Sigma}_{34,34}^{-1}}{\partial \sigma_{a b} \partial \sigma_{c d}} \boldsymbol{\sigma}_{34,1}, \\
\frac{\partial^{2} \psi_{2 \mathrm{~B}}}{\partial \sigma_{a b} \partial \sigma_{c d}}= & \sum \frac{\partial \boldsymbol{\sigma}_{35,2}^{\prime}}{\partial \sigma_{a b}} \frac{\partial \boldsymbol{\Sigma}_{35,35}^{-1}}{\partial \sigma_{c d}} \boldsymbol{\sigma}_{35,2}+\boldsymbol{\sigma}_{35,2}^{\prime} \frac{\partial^{2} \boldsymbol{\Sigma}_{35,35}^{-1}}{\partial \sigma_{a b} \partial \sigma_{c d}} \boldsymbol{\sigma}_{35,2}, \\
\frac{\partial^{2} \psi_{3 \mathrm{~B}}}{\partial \sigma_{a b} \partial \sigma_{c d}}= & \sum \frac{\partial \boldsymbol{\sigma}_{34,1}^{\prime}}{\partial \sigma_{a b}} \frac{\partial \boldsymbol{\Sigma}_{34,34}^{-1}}{\partial \sigma_{c d}} \boldsymbol{\sigma}_{34,2}+\boldsymbol{\sigma}_{34,1}^{\prime} \frac{\partial^{2} \boldsymbol{\Sigma}_{34,34}^{-1}}{\partial \sigma_{a b} \partial \sigma_{c d}} \boldsymbol{\sigma}_{34,2}, \\
\frac{\partial^{2} \psi_{3 \mathrm{C}}}{\partial \sigma_{a b} \partial \sigma_{c d}}= & \sum \frac{\partial \boldsymbol{\sigma}_{35,1}^{\prime}}{\partial \sigma_{a b}} \frac{\partial \boldsymbol{\Sigma}_{35,35}^{-1}}{\partial \sigma_{c d}} \boldsymbol{\sigma}_{35,2}+\boldsymbol{\sigma}_{35,1}^{\prime} \frac{\partial^{2} \boldsymbol{\Sigma}_{35,35}^{-1}}{\partial \sigma_{a b} \partial \sigma_{c d}} \boldsymbol{\sigma}_{35,2}, \\
\frac{\partial^{2} \psi_{3 \mathrm{D}}}{\partial \sigma_{a b} \partial \sigma_{c d}}= & \sum \frac{{ }_{5} P_{2}=}{20} \frac{\partial \boldsymbol{\sigma}_{34,1}^{\prime}}{\partial \sigma_{a b}} \frac{\partial \boldsymbol{\Sigma}_{34,34}^{-1}}{\partial \sigma_{c d}} \boldsymbol{\Sigma}_{34,35} \boldsymbol{\Sigma}_{35,35}^{-1} \boldsymbol{\sigma}_{35,2} \\
& +\boldsymbol{\sigma}_{34,1}^{\prime} \frac{\partial^{2} \boldsymbol{\Sigma}_{34,34}^{-1}}{\partial \sigma_{a b} \partial \sigma_{c d}} \boldsymbol{\Sigma}_{34,35} \boldsymbol{\Sigma}_{35,35}^{-1} \boldsymbol{\sigma}_{35,2}+\boldsymbol{\sigma}_{34,1}^{\prime} \boldsymbol{\Sigma}_{34,34}^{-1} \boldsymbol{\Sigma}_{34,35} \frac{\partial^{2} \boldsymbol{\Sigma}_{35,35}^{-1}}{\partial \sigma_{a b} \partial \sigma_{c d}} \boldsymbol{\sigma}_{35,2}, \\
\frac{\partial^{3} \psi_{1 \mathrm{~B}}}{\partial \sigma_{a b} \partial \sigma_{c d} \partial \sigma_{e f}}= & \sum \frac{\partial \boldsymbol{\sigma}_{34,1}^{\prime}}{\partial \sigma_{a b}} \frac{\partial \boldsymbol{\Sigma}_{34,34}^{-1}}{\partial \sigma_{c d}} \frac{\partial \boldsymbol{\sigma}_{34,1}}{\partial \sigma_{e f}}+\sum^{6} \frac{\partial \boldsymbol{\sigma}_{34,1}^{\prime}}{\partial \sigma_{a b}} \frac{\partial^{2} \boldsymbol{\Sigma}_{34,34}^{-1}}{\partial \sigma_{c d} \partial \sigma_{e f}} \boldsymbol{\sigma}_{34,1} \\
& +\boldsymbol{\sigma}_{34,1}^{\prime} \frac{\partial^{3} \boldsymbol{\Sigma}_{34,34}^{-1}}{\partial \sigma_{a b} \partial \sigma_{c d} \partial \sigma_{e f}} \boldsymbol{\sigma}_{34,1},
\end{aligned}
$$




$$
\begin{aligned}
& \frac{\partial^{3} \psi_{2 \mathrm{~B}}}{\partial \sigma_{a b} \partial \sigma_{c d} \partial \sigma_{e f}}=\sum^{6} \frac{\partial \boldsymbol{\sigma}_{35,2}^{\prime}}{\partial \sigma_{a b}} \frac{\partial \boldsymbol{\Sigma}_{35,35}^{-1}}{\partial \sigma_{c d}} \frac{\partial \boldsymbol{\sigma}_{35,2}}{\partial \sigma_{e f}}+\sum^{6} \frac{\partial \boldsymbol{\sigma}_{35,2}^{\prime}}{\partial \sigma_{a b}} \frac{\partial^{2} \boldsymbol{\Sigma}_{35,35}^{-1}}{\partial \sigma_{c d} \partial \sigma_{e f}} \boldsymbol{\sigma}_{35,2} \\
& +\boldsymbol{\sigma}_{35,2}^{\prime} \frac{\partial^{3} \boldsymbol{\Sigma}_{35,35}^{-1}}{\partial \sigma_{a b} \partial \sigma_{c d} \partial \sigma_{e f}} \boldsymbol{\sigma}_{35,2}, \\
& \frac{\partial^{3} \psi_{3 \mathrm{~B}}}{\partial \sigma_{a b} \partial \sigma_{c d} \partial \sigma_{e f}}=\sum^{6} \frac{\partial \boldsymbol{\sigma}_{34,1}^{\prime}}{\partial \sigma_{a b}} \frac{\partial \boldsymbol{\Sigma}_{34,34}^{-1}}{\partial \sigma_{c d}} \frac{\partial \boldsymbol{\sigma}_{34,2}}{\partial \sigma_{e f}}+\sum^{6} \frac{\partial \boldsymbol{\sigma}_{34,1}^{\prime}}{\partial \sigma_{a b}} \frac{\partial^{2} \boldsymbol{\Sigma}_{34,34}^{-1}}{\partial \sigma_{c d} \partial \sigma_{e f}} \boldsymbol{\sigma}_{34,2} \\
& +\boldsymbol{\sigma}_{34,1}^{\prime} \frac{\partial^{3} \boldsymbol{\Sigma}_{34,34}^{-1}}{\partial \sigma_{a b} \partial \sigma_{c d} \partial \sigma_{e f}} \boldsymbol{\sigma}_{34,2}, \\
& \frac{\partial^{3} \psi_{3 \mathrm{C}}}{\partial \sigma_{a b} \partial \sigma_{c d} \partial \sigma_{e f}}=\sum^{6} \frac{\partial \boldsymbol{\sigma}_{35,1}^{\prime}}{\partial \sigma_{a b}} \frac{\partial \boldsymbol{\Sigma}_{35,35}^{-1}}{\partial \sigma_{c d}} \frac{\partial \boldsymbol{\sigma}_{35,2}}{\partial \sigma_{e f}}+\sum^{6} \frac{\partial \boldsymbol{\sigma}_{35,1}^{\prime}}{\partial \sigma_{a b}} \frac{\partial^{2} \boldsymbol{\Sigma}_{35,35}^{-1}}{\partial \sigma_{c d} \partial \sigma_{e f}} \boldsymbol{\sigma}_{35,2} \\
& +\boldsymbol{\sigma}_{35,1}^{\prime} \frac{\partial^{3} \boldsymbol{\Sigma}_{35,35}^{-1}}{\partial \sigma_{a b} \partial \sigma_{c d} \partial \sigma_{e f}} \boldsymbol{\sigma}_{35,2}, \\
& \frac{\partial^{3} \psi_{3 \mathrm{D}}}{\partial \sigma_{a b} \partial \sigma_{c d} \partial \sigma_{e f}}=\sum^{5} \sum^{P_{3}=60} \frac{\partial \boldsymbol{\sigma}_{34,1}^{\prime}}{\partial \sigma_{a b}} \frac{\partial \boldsymbol{\Sigma}_{34,34}^{-1}}{\partial \sigma_{c d}} \frac{\partial \boldsymbol{\Sigma}_{34,35}}{\partial \sigma_{e f}} \boldsymbol{\Sigma}_{35,35}^{-1} \boldsymbol{\sigma}_{35,2} \\
& +\sum^{12}\left(\frac{\partial \boldsymbol{\sigma}_{34,1}^{\prime}}{\partial \sigma_{a b}} \frac{\partial^{2} \boldsymbol{\Sigma}_{34,34}^{-1}}{\partial \sigma_{c d} \partial \sigma_{e f}} \boldsymbol{\Sigma}_{34,35} \boldsymbol{\Sigma}_{35,35}^{-1} \boldsymbol{\sigma}_{35,2}\right. \\
& \left.+\frac{\partial \boldsymbol{\sigma}_{34,1}^{\prime}}{\partial \sigma_{a b}} \boldsymbol{\Sigma}_{34,34}^{-1} \boldsymbol{\Sigma}_{34,35} \frac{\partial^{2} \boldsymbol{\Sigma}_{35,35}^{-1}}{\partial \sigma_{c d} \partial \sigma_{e f}} \boldsymbol{\sigma}_{35,2}\right) \\
& +\boldsymbol{\sigma}_{34,1}^{\prime} \frac{\partial^{3} \boldsymbol{\Sigma}_{34,34}^{-1}}{\partial \sigma_{a b} \partial \sigma_{c d} \partial \sigma_{e f}} \boldsymbol{\Sigma}_{34,35} \boldsymbol{\Sigma}_{35,35}^{-1} \boldsymbol{\sigma}_{35,2} \\
& +\boldsymbol{\sigma}_{34,1}^{\prime} \boldsymbol{\Sigma}_{34,34}^{-1} \boldsymbol{\Sigma}_{34,35} \frac{\partial^{3} \boldsymbol{\Sigma}_{35,35}^{-1}}{\partial \sigma_{a b} \partial \sigma_{c d} \partial \sigma_{e f}} \boldsymbol{\sigma}_{35,2} \\
& (p \geq a \geq b \geq 1 ; p \geq c \geq d \geq 1 ; p \geq e \geq f \geq 1) \text {. }
\end{aligned}
$$

The nonzero partial derivatives of $\boldsymbol{\sigma}_{34,1}, \boldsymbol{\Sigma}_{34,34}^{-1}, \boldsymbol{\Sigma}_{34,35}$ and $\boldsymbol{\sigma}_{35,2}$ with respect to $\sigma_{a b}$ 's, in form, are

$$
\begin{aligned}
& \frac{\partial \boldsymbol{\sigma}_{34,1}}{\partial \boldsymbol{\sigma}_{34,1}^{\prime}}=\mathbf{I}_{p_{3}+p_{4}} \\
& \frac{\partial\left(\boldsymbol{\Sigma}_{34,34}^{-1}\right)_{i j}}{\partial\left(\boldsymbol{\Sigma}_{34,34}\right)_{a b}}=-\frac{2-\delta_{a b}}{2}\left\{\left(\boldsymbol{\Sigma}_{34,34}^{-1}\right)_{i a}\left(\boldsymbol{\Sigma}_{34,34}^{-1}\right)_{j b}+\left(\boldsymbol{\Sigma}_{34,34}^{-1}\right)_{i b}\left(\boldsymbol{\Sigma}_{34,34}^{-1}\right)_{j a}\right\}, \\
& \frac{\partial^{2}\left(\boldsymbol{\Sigma}_{34,34}^{-1}\right)_{i j}}{\partial\left(\boldsymbol{\Sigma}_{34,34}\right)_{a b} \partial\left(\boldsymbol{\Sigma}_{34,34}\right)_{c d}}=\frac{1}{4}\left(2-\delta_{a b}\right)\left(2-\delta_{c d}\right) \sum^{8}\left(\boldsymbol{\Sigma}_{34,34}^{-1}\right)_{i c}\left(\boldsymbol{\Sigma}_{34,34}^{-1}\right)_{d a}\left(\boldsymbol{\Sigma}_{34,34}^{-1}\right)_{b j}, \\
& \frac{\partial^{3}\left(\boldsymbol{\Sigma}_{34,34}^{-1}\right)_{i j}}{\partial\left(\boldsymbol{\Sigma}_{34,34}\right)_{a b} \partial\left(\boldsymbol{\Sigma}_{34,34}\right)_{c d} \partial\left(\boldsymbol{\Sigma}_{34,34}\right)_{e f}}=-\frac{1}{8}\left(2-\delta_{a b}\right)\left(2-\delta_{c d}\right)\left(2-\delta_{e f}\right) \\
& \times \sum^{48}\left(\boldsymbol{\Sigma}_{34,34}^{-1}\right)_{i e}\left(\boldsymbol{\Sigma}_{34,34}^{-1}\right)_{f c}\left(\boldsymbol{\Sigma}_{34,34}^{-1}\right)_{d a}\left(\boldsymbol{\Sigma}_{34,34}^{-1}\right)_{b j}
\end{aligned}
$$

$\left(i, j=1, \ldots, p_{3}+p_{4} ; p_{3}+p_{4} \geq a \geq b \geq 1 ; p_{3}+p_{4} \geq c \geq d \geq 1 ; p_{3}+p_{4} \geq e \geq f \geq 1\right)$, 


$$
\begin{aligned}
& \frac{\partial \boldsymbol{\Sigma}_{34,35}}{\partial\left(\boldsymbol{\Sigma}_{34,35}\right)_{a b}}=\frac{2-\delta_{a b}}{2}\left(\mathbf{E}_{a b}+\mathbf{E}_{b a}\right)\left(p_{3} \geq a \geq b \geq 1\right), \\
& \frac{\partial \boldsymbol{\Sigma}_{34,35}}{\partial\left(\boldsymbol{\Sigma}_{34,35}\right)_{p_{3}+a, b}}=\mathbf{E}_{p_{3}+a, b} \quad\left(a=1, \ldots, p_{4} ; b=1, \ldots, p_{3}\right), \\
& \frac{\partial \boldsymbol{\Sigma}_{34,35}}{\partial\left(\boldsymbol{\Sigma}_{34,35}\right)_{a, p_{3}+b}}=\mathbf{E}_{a, p_{3}+b} \quad\left(a=1, \ldots, p_{3} ; b=1, \ldots, p_{5}\right), \quad \frac{\partial \boldsymbol{\sigma}_{35,2}}{\partial \boldsymbol{\sigma}_{35,2}^{\prime}}=\mathbf{I}_{p_{3}+p_{5}},
\end{aligned}
$$

where $\mathbf{I}_{k}$ is the $k \times k$ identity matrix, $\delta_{a b}$ is the Kronecker delta, $\mathbf{E}_{a b}$ is the matrix of an appropriate size, where the $(a, b)$ th element is 1 with other elements being 0 . The partial derivatives of $\boldsymbol{\Sigma}_{35,35}^{-1}$, in form, are given from those of $\boldsymbol{\Sigma}_{34,34}^{-1}$ by replacing $\boldsymbol{\Sigma}_{34,34}$ and $p_{4}$ with $\boldsymbol{\Sigma}_{35,35}$ and $p_{5}$, respectively.

\section{B. The partial derivatives of $\hat{\rho}_{\mathrm{M} 1}^{2}$ with respect to $\mathrm{s}$}

Rewrite $\rho_{\mathrm{M} 1}^{2}$ as $\rho_{\mathrm{M} 1}^{2}=1-\exp \left(-l^{*}\right), l^{*}=-\ln |\boldsymbol{\Psi}|+\ln \left|\boldsymbol{\Psi}_{11}\right|+\ln \left|\boldsymbol{\Psi}_{22}\right|$, where $l^{*}$ is the population value of $-(2 / n)$ times the $\log$ likelihood ratio. Then, in the first step,

$$
\begin{aligned}
& \frac{\partial \rho_{\mathrm{M} 1}^{2}}{\partial \sigma_{a b}}=\exp \left(-l^{*}\right) \frac{\partial l^{*}}{\partial \sigma_{a b}}=\left(1-\rho_{\mathrm{M} 1}^{2}\right) \frac{\partial l^{*}}{\partial \sigma_{a b}} \\
& \frac{\partial^{2} \rho_{\mathrm{M} 1}^{2}}{\partial \sigma_{a b} \partial \sigma_{c d}}=\left(1-\rho_{\mathrm{M} 1}^{2}\right)\left(-\frac{\partial l^{*}}{\partial \sigma_{a b}} \frac{\partial l^{*}}{\partial \sigma_{c d}}+\frac{\partial^{2} l^{*}}{\partial \sigma_{a b} \partial \sigma_{c d}}\right), \\
& \frac{\partial^{3} \rho_{\mathrm{M} 1}^{2}}{\partial \sigma_{a b} \partial \sigma_{c d} \partial \sigma_{e f}}=\left(1-\rho_{\mathrm{M} 1}^{2}\right)\left(\frac{\partial l^{*}}{\partial \sigma_{a b}} \frac{\partial l^{*}}{\partial \sigma_{c d}} \frac{\partial l^{*}}{\partial \sigma_{e f}}-\sum \frac{3}{\partial \sigma_{a b}} \frac{\partial^{2} l^{*}}{\partial \sigma_{c d} \partial \sigma_{e f}}+\frac{\partial^{3} l^{*}}{\partial \sigma_{a b} \partial \sigma_{c d} \partial \sigma_{e f}}\right) \\
& (p \geq a \geq b \geq 1 ; p \geq c \geq d \geq 1 ; p \geq e \geq f \geq 1),
\end{aligned}
$$

where $p=\sum_{i=1}^{5} p_{i}$. The partial derivatives of $\hat{l}^{*}$ with respect to $s_{s b}$ 's are given from

$$
\begin{aligned}
& \frac{\partial \ln |\boldsymbol{\Psi}|}{\partial \sigma_{a b}}=\operatorname{tr}\left(\boldsymbol{\Psi}^{-1} \frac{\partial \boldsymbol{\Psi}}{\partial \sigma_{a b}}\right), \\
& \frac{\partial^{2} \ln |\boldsymbol{\Psi}|}{\partial \sigma_{a b} \partial \sigma_{c d}}=\operatorname{tr}\left(-\boldsymbol{\Psi}^{-1} \frac{\partial \boldsymbol{\Psi}}{\partial \sigma_{a b}} \boldsymbol{\Psi}^{-1} \frac{\partial \boldsymbol{\Psi}}{\partial \sigma_{c d}}+\boldsymbol{\Psi}^{-1} \frac{\partial^{2} \boldsymbol{\Psi}}{\partial \sigma_{a b} \partial \sigma_{c d}}\right), \\
& \frac{\partial^{3} \ln |\boldsymbol{\Psi}|}{\partial \sigma_{a b} \partial \sigma_{c d} \partial \sigma_{e f}}=\operatorname{tr}\left(2 \boldsymbol{\Psi}^{-1} \frac{\partial \boldsymbol{\Psi}}{\partial \sigma_{a b}} \boldsymbol{\Psi}^{-1} \frac{\partial \boldsymbol{\Psi}}{\partial \sigma_{c d}} \boldsymbol{\Psi}^{-1} \frac{\partial \boldsymbol{\Psi}}{\partial \sigma_{e f}}\right. \\
& \left.\quad-\sum^{3} \boldsymbol{\Psi}^{-1} \frac{\partial \boldsymbol{\Psi}}{\partial \sigma_{a b}} \boldsymbol{\Psi}^{-1} \frac{\partial^{2} \boldsymbol{\Psi}}{\partial \sigma_{c d} \partial \sigma_{e f}}+\boldsymbol{\Psi}^{-1} \frac{\partial^{3} \boldsymbol{\Psi}}{\partial \sigma_{a b} \partial \sigma_{c d} \partial \sigma_{e f}}\right) \\
& (p \geq a \geq b \geq 1 ; p \geq c \geq d \geq 1 ; p \geq e \geq f \geq 1),
\end{aligned}
$$

where the partial derivatives of $\boldsymbol{\Psi}$ with respect to $\sigma_{a b}$ 's, in form, are given in a manner similar to those of $\psi_{1}, \psi_{2}$ and $\psi_{3}$ in Subsection 1.3 of this appendix with the following definitions:

$$
\begin{aligned}
(\boldsymbol{\Psi})_{1_{i} 1_{j}}= & \left(\boldsymbol{\Psi}_{11}\right)_{i j}=\sigma_{1_{i} 1_{j}}-\boldsymbol{\sigma}_{34,1_{i}}^{\prime} \boldsymbol{\Sigma}_{34,34}^{-1} \boldsymbol{\sigma}_{34,1_{j}} \quad\left(i, j=1, \ldots, p_{1}\right), \\
(\boldsymbol{\Psi})_{1_{i} 2_{j}}= & \left(\boldsymbol{\Psi}_{12}\right)_{i j}=\sigma_{1_{i} 2_{j}}-\boldsymbol{\sigma}_{34,1_{i}}^{\prime} \boldsymbol{\Sigma}_{34,34}^{-1} \boldsymbol{\sigma}_{34,2_{j}}-\boldsymbol{\sigma}_{35,1_{i}}^{\prime} \boldsymbol{\Sigma}_{35,35}^{-1} \boldsymbol{\sigma}_{35,2_{j}} \\
& +\boldsymbol{\sigma}_{34,1_{i}}^{\prime} \boldsymbol{\Sigma}_{34,34}^{-1} \boldsymbol{\Sigma}_{34,35} \boldsymbol{\Sigma}_{35,35}^{-1} \boldsymbol{\sigma}_{35,2_{j}} \quad\left(i=1, \ldots, p_{1} ; j=1, \ldots, p_{2}\right),
\end{aligned}
$$




$$
(\boldsymbol{\Psi})_{2_{i} 2_{j}}=\left(\boldsymbol{\Psi}_{22}\right)_{i j}=\sigma_{2_{i} 2_{j}}-\boldsymbol{\sigma}_{35,2_{i}}^{\prime} \boldsymbol{\Sigma}_{35,35}^{-1} \boldsymbol{\sigma}_{35,2_{j}} \quad\left(i, j=1, \ldots, p_{2}\right),
$$

where $1_{i}=i\left(i=1, \ldots, p_{1}\right)$ and $2_{i}=p_{1}+i\left(i=1, \ldots, p_{2}\right)$.

The partial derivatives of $\ln \left|\boldsymbol{\Psi}_{11}\right|$ and $\ln \left|\boldsymbol{\Psi}_{22}\right|$ with respect to $\sigma_{a b}$ 's, in form, are similarly obtained.

\section{The partial derivatives of $\hat{\rho}_{\mathrm{M} 2}^{2}$ with respect to $\mathrm{s}$}

For convenience, $\boldsymbol{\Psi}_{11}^{-1} \boldsymbol{\Psi}_{12} \boldsymbol{\Psi}_{22}^{-1} \boldsymbol{\Psi}_{21}$ is seen as the product of $\boldsymbol{\Psi}_{11}^{-1} \boldsymbol{\Psi}_{12}$ and $\boldsymbol{\Psi}_{22}^{-1} \boldsymbol{\Psi}_{21}$. Then, in the first step,

$$
\begin{aligned}
& \frac{\partial \rho_{\mathrm{M} 2}^{2}}{\partial \sigma_{a b}}=c^{-1} \operatorname{tr}\left(\frac{\partial \boldsymbol{\Psi}_{11}^{-1} \boldsymbol{\Psi}_{12}}{\partial \sigma_{a b}} \boldsymbol{\Psi}_{22}^{-1} \boldsymbol{\Psi}_{21}+\boldsymbol{\Psi}_{11}^{-1} \boldsymbol{\Psi}_{12} \frac{\partial \boldsymbol{\Psi}_{22}^{-1} \boldsymbol{\Psi}_{21}}{\partial \sigma_{a b}}\right), \\
& \frac{\partial^{2} \rho_{\mathrm{M} 2}^{2}}{\partial \sigma_{a b} \partial \sigma_{c d}}=c^{-1} \operatorname{tr}\left(\frac{\partial \boldsymbol{\Psi}_{11}^{-1} \boldsymbol{\Psi}_{12}}{\partial \sigma_{a b}} \frac{\partial \boldsymbol{\Psi}_{22}^{-1} \boldsymbol{\Psi}_{21}}{\partial \sigma_{c d}}+\frac{\partial \boldsymbol{\Psi}_{11}^{-1} \boldsymbol{\Psi}_{12}}{\partial \sigma_{c d}} \frac{\partial \boldsymbol{\Psi}_{22}^{-1} \boldsymbol{\Psi}_{21}}{\partial \sigma_{a b}}\right. \\
& \left.+\frac{\partial^{2} \boldsymbol{\Psi}_{11}^{-1} \boldsymbol{\Psi}_{12}}{\partial \sigma_{a b} \partial \sigma_{c d}} \boldsymbol{\Psi}_{22}^{-1} \boldsymbol{\Psi}_{21}+\boldsymbol{\Psi}_{11}^{-1} \boldsymbol{\Psi}_{12} \frac{\partial^{2} \boldsymbol{\Psi}_{22}^{-1} \boldsymbol{\Psi}_{21}}{\partial \sigma_{a b} \partial \sigma_{c d}}\right) \\
& \frac{\partial^{3} \rho_{\mathrm{M} 2}^{2}}{\partial \sigma_{a b} \partial \sigma_{c d} \partial \sigma_{e f}}=\operatorname{tr}\left(\sum^{6} \frac{\partial \boldsymbol{\Psi}_{11}^{-1} \boldsymbol{\Psi}_{12}}{\partial \sigma_{a b}} \frac{\partial^{2} \boldsymbol{\Psi}_{22}^{-1} \boldsymbol{\Psi}_{21}}{\partial \sigma_{c d} \partial \sigma_{e f}}+\frac{\partial^{3} \boldsymbol{\Psi}_{11}^{-1} \boldsymbol{\Psi}_{12}}{\partial \sigma_{a b} \partial \sigma_{c d} \partial \sigma_{e f}} \boldsymbol{\Psi}_{22}^{-1} \mathbf{\Psi}_{21}\right. \\
& \left.+\boldsymbol{\Psi}_{11}^{-1} \boldsymbol{\Psi}_{12} \frac{\partial^{3} \boldsymbol{\Psi}_{22}^{-1} \boldsymbol{\Psi}_{21}}{\partial \sigma_{a b} \partial \sigma_{c d} \partial \sigma_{e f}}\right), \\
& (p \geq a \geq b \geq 1 ; p \geq c \geq d \geq 1 ; p \geq e \geq f \geq 1) \text {. }
\end{aligned}
$$

In the above expressions, the partial derivatives of $\boldsymbol{\Psi}_{11}^{-1} \boldsymbol{\Psi}_{12}$ and $\boldsymbol{\Psi}_{22}^{-1} \boldsymbol{\Psi}_{21}$ with respect to $\sigma_{a b}$ 's, in form, are

$$
\begin{aligned}
& \frac{\partial \boldsymbol{\Psi}_{11}^{-1} \boldsymbol{\Psi}_{12}}{\partial\left(\boldsymbol{\Psi}_{11}\right)_{a b}}=-\frac{2-\delta_{a b}}{2} \boldsymbol{\Psi}_{11}^{-1}\left(\mathbf{E}_{a b}+\mathbf{E}_{b a}\right) \boldsymbol{\Psi}_{11}^{-1} \boldsymbol{\Psi}_{12} \\
& =-\frac{2-\delta_{a b}}{2}\left\{\left(\boldsymbol{\Psi}_{11}^{-1}\right)_{\cdot a}\left(\boldsymbol{\Psi}_{11}^{-1} \boldsymbol{\Psi}_{12}\right)_{b} .+\left(\boldsymbol{\Psi}_{11}^{-1}\right)_{\cdot b}\left(\boldsymbol{\Psi}_{11}^{-1} \boldsymbol{\Psi}_{12}\right)_{a} \cdot\right\} \quad\left(p_{1} \geq a \geq b \geq 1\right), \\
& \frac{\partial\left(\boldsymbol{\Psi}_{11}^{-1} \mathbf{\Psi}_{12}\right)_{\cdot b}}{\partial\left(\mathbf{\Psi}_{12}\right)_{a b}}=\left(\mathbf{\Psi}_{11}^{-1}\right)_{\cdot a} \quad\left(a=1, \ldots, p_{1} ; b=1, \ldots, p_{2}\right), \\
& \frac{\partial^{2} \boldsymbol{\Psi}_{11}^{-1} \boldsymbol{\Psi}_{12}}{\partial\left(\boldsymbol{\Psi}_{11}\right)_{a b} \partial\left(\boldsymbol{\Psi}_{11}\right)_{c d}}=\frac{1}{4}\left(2-\delta_{a b}\right)\left(2-\delta_{c d}\right) \sum^{8}\left(\boldsymbol{\Psi}_{11}^{-1}\right)_{\cdot a}\left(\boldsymbol{\Psi}_{11}^{-1}\right)_{b c}\left(\boldsymbol{\Psi}_{11}^{-1} \boldsymbol{\Psi}_{12}\right)_{d} . \\
& \left(p_{1} \geq a \geq b \geq 1 ; p_{1} \geq c \geq d \geq 1\right), \\
& \frac{\partial^{2}\left(\boldsymbol{\Psi}_{11}^{-1} \boldsymbol{\Psi}_{12}\right)_{\cdot b}}{\partial\left(\boldsymbol{\Psi}_{12}\right)_{a b} \partial\left(\boldsymbol{\Psi}_{11}\right)_{c d}}=-\frac{1}{2}\left(2-\delta_{c d}\right)\left\{\boldsymbol{\Psi}_{11}^{-1}\left(\mathbf{E}_{c d}+\mathbf{E}_{d c}\right) \boldsymbol{\Psi}_{11}^{-1}\right\}_{\cdot a} \\
& =-\frac{2-\delta_{c d}}{2}\left\{\left(\Psi_{11}^{-1}\right)_{\cdot c}\left(\mathbf{\Psi}_{11}^{-1}\right)_{d a}+\left(\mathbf{\Psi}_{11}^{-1}\right)_{\cdot d}\left(\mathbf{\Psi}_{11}^{-1}\right)_{c a}\right\} \\
& \left(a=1, \ldots, p_{1} ; b=1, \ldots, p_{2} ; p_{1} \geq c \geq d \geq 1\right), \\
& \frac{\partial^{3} \boldsymbol{\Psi}_{11}^{-1} \boldsymbol{\Psi}_{12}}{\partial\left(\boldsymbol{\Psi}_{11}\right)_{a b} \partial\left(\boldsymbol{\Psi}_{11}\right)_{c d} \partial\left(\boldsymbol{\Psi}_{11}\right)_{e f}}=-\frac{1}{8}\left(2-\delta_{a b}\right)\left(2-\delta_{c d}\right)\left(2-\delta_{e f}\right) \sum^{48}\left(\boldsymbol{\Psi}_{11}^{-1}\right)_{\cdot a}\left(\boldsymbol{\Psi}_{11}^{-1}\right)_{b c}
\end{aligned}
$$




$$
\begin{aligned}
& \times\left(\boldsymbol{\Psi}_{11}^{-1}\right)_{d e}\left(\boldsymbol{\Psi}_{11}^{-1} \boldsymbol{\Psi}_{12}\right)_{f} . \\
&\left(p_{1} \geq a \geq b \geq 1 ; p_{1} \geq c \geq d \geq\right.\left.1 ; p_{1} \geq e \geq f \geq 1\right), \\
& \frac{\partial^{3}\left(\boldsymbol{\Psi}_{11}^{-1} \mathbf{\Psi}_{12}\right)_{\cdot b}}{\partial\left(\boldsymbol{\Psi}_{12}\right)_{a b} \partial\left(\boldsymbol{\Psi}_{11}\right)_{c d} \partial\left(\boldsymbol{\Psi}_{11}\right)_{e f}}= \frac{1}{4}\left(2-\delta_{c d}\right)\left(2-\delta_{e f}\right) \sum^{8}\left(\boldsymbol{\Psi}_{11}^{-1}\right)_{\cdot c}\left(\mathbf{\Psi}_{11}^{-1}\right)_{d e}\left(\mathbf{\Psi}_{11}^{-1}\right)_{f a} \\
&\left(a=1, \ldots, p_{1} ; b=1, \ldots, p_{2} ; p_{1} \geq c \geq d \geq 1 ; p_{1} \geq e \geq f \geq 1\right),
\end{aligned}
$$

where $(\cdot)_{a}$ and $(\cdot)_{b}$. denote the $a$-th column and $b$-th row of the argument matrices, respectively. The partial derivatives of $\boldsymbol{\Psi}_{22}^{-1} \boldsymbol{\Psi}_{21}$ with respect to $\sigma_{a b}$ 's, in form, are given by replacing $\boldsymbol{\Psi}_{11}, \mathbf{\Psi}_{12}, p_{1}$ and $p_{2}$ in the above results by $\mathbf{\Psi}_{22}, \mathbf{\Psi}_{21}, p_{2}$ and $p_{1}$, respectively.

\section{REFERENCES}

Akahira, M., \& Torigoe, N. (1998). A new higher order approximation to a percentage point of the distribution of the sample correlation coefficient. Journal of Japan Statistical Society, $28,45-57$.

Anderson, T.W. (2003). An introduction to multivariate statistical analysis (3rd ed.). New York: Wiley.

Boik, R.J. (1998). A local parameterization of orthogonal and semi-orthogonal matrices with applications. Journal of Multivariate Analysis, 67, 244-276.

Boik, R.J., \& Haaland, B. (2006). Second-order accurate inference on simple, partial, and multiple correlations. Journal of Modern Applied Statistical Methods, 5, 283-308.

Browne, M.W., \& Shapiro, A. (1986). The asymptotic covariance matrix of sample correlation coefficients under general conditions. Linear Algebra and Its Applications, 82, 169-176.

Cohen, J. (1982). Set correlation as a general multivariate data-analytic method. Multivariate Behavioral Research, 17, 301-341.

Cohen, J., Cohen, P., West, S.G., \& Aiken, L.S. (2003). Applied multiple regression/correlation analysis for the behavioral sciences (3rd ed.). Mahwah, NJ: LEA.

Cook, M.B. (1951). Two applications of bivariate $k$-statistics. Biometrika, 38, 368-376.

Cramer, E.M., \& Nicewander, W.A. (1979). Some symmetric, invariant measures of multivariate association. Psychometrika, 44, 43-54.

Ezekiel, M. (1941). Methods of correlation analysis (2nd ed.). New York: Wiley.

Fisher, R.A. (1915). Frequency distribution of the values of the correlation coefficient in samples from an indefinitely large population. Biometrika, 10, 507-521.

Fisher, R.A. (1924). The distribution of the partial correlation coefficient. Metron, 3, 329-332.

Fujikoshi, Y. (1980). Asymptotic expansions for the distributions of the sample roots under nonnormality. Biometrika, 67, 45-51.

Girshick, M.A. (1939). On the sampling theory of roots of determinantal equations. Annals of Mathematical Statistics, 10, 203-224.

Hall, P. (1992). On the removal of skewness by transformation. Journal of the Royal Statistical Society, B, 54, 221-228.

Hooper, J.M. (1959). Simultaneous equations and canonical correlation theory. Econometrica, 27, $245-256$.

Hooper, J.M. (1962). Partial trace correlations. Econometrica, 30, 324-331.

Hotelling, H. (1936). Relations between two sets of variates. Biometrika, 28, 321-377.

Hotelling, H. (1953). New light on the correlation coefficient and its transforms. (with discussion) Journal of the Royal Statistical Society, Series B, 15, 193-232.

Hsu, P.L. (1949). The limiting distribution of functions of sample means and application to 
testing hypotheses. In J. Neyman (Ed.), Proceedings of the First Berkeley Symposium on Mathematical Statistics and Probability (pp.359-402). Berkeley: University of California Press.

Isserlis, L. (1916). On certain probable errors and correlation coefficients of multiple frequency distributions with skew regression. Biometrika, 11, 185-190

Kollo, T., \& Ruul, K. (2003). Approximations to the distribution of the sample correlation matrix. Journal of Multivariate Analysis, 85, 318-334.

Kollo, T., \& von Rosen, D. (2005). Advanced multivariate statistics and matrices. Dordrecht, The Netherlands: Springer.

Konishi, S. (1978). An approximation to the distribution of the sample correlation coefficient. Biometrika, 65, 654-656.

Konishi, S. (1979a). Asymptotic expansions for the distributions of functions of a correlation matrix. Journal of Multivariate Analysis, 9, 259-266.

Konishi, S. (1979b). Asymptotic expansions for the distributions of statistics based on the sample correlation matrix in principal component analysis. Hiroshima Mathematical Journal, 9, 647-700.

Lee, S.-Y. (1978). Generalizations of the partial, part and bipartial canonical correlation analysis. Psychometrika, 43, 427-431.

Muirhead, R.J. (1982). Aspects of multivariate statistical theory. New York: Wiley.

Nakagawa, S., \& Niki, N. (1992). Distribution of the sample correlation coefficient for nonnormal populations. Journal of the Japanese Society of Computational Statistics, 5, 1-19.

Nel, D.G. (1985). A matrix derivation of the asymptotic covariance matrix of sample correlation coefficients. Linear Algebra and Its Applications, 67, 137-145.

Neudecker, H. (1996). The asymptotic variance matrices of the sample correlation matrix in elliptical and normal situations and their proportionality. Linear Algebra and Its Applications, 237-238, 127-132.

Neudecker, H., \& Wesselman, A.M. (1990). The asymptotic variance matrix of the sample correlation matrix. Linear Algebra and Its Applications, 127, 589-599.

Niki, N., \& Konishi, S. (1984). Higher order asymptotic expansions for the distribution of the sample correlation coefficient. Communications in Statistics-Simulation and Computation, 13, 169-182.

Ogasawara, H. (2004). Asymptotic biases in exploratory factor analysis and structural equation modeling. Psychometrika, 69, 235-256.

Ogasawara, H. (2006a). Asymptotic expansion of the sample correlation coefficient under nonnormality. Computational Statistics and Data Analysis, 50, 891-910.

Ogasawara, H. (2006b). Asymptotic expansion and conditional robustness for the sample multiple correlation coefficient under nonnormality. Communications in Statistics: Simulation and Computation, 35, 177-199.

Ogasawara, H. (2007). Asymptotic expansion of the distributions of the estimators in factor analysis under nonnormality. British Journal of Mathematical and Statistical Psychology, 60, 395-420.

Olkin, I., \& Siotani, M. (1976). Asymptotic distribution of functions of a correlation matrix. In S. Ikeda et al. (Eds.) Essays in provability and statistics: A volume in honor of Professor Junjiro Ogawa (pp. 235-251). Tokyo: Shinko Tsusho.

Pearson, K., \& Filon, L.N.G. (1898). Mathematical contributions to the theory of evolution.-IV. On the probable errors of frequency constants and on the influence of random selection on variation and correlation. Philosophical Transactions of the Royal Society of London. Series A, Mathematical and Physical Sciences, 191, 229-311.

Rozeboom, W.W. (1965). Linear correlations between sets of variables. Psychometrika, 30, 57-71. Steiger, J.H., \& Browne, M.W. (1984). The comparison of interdependent correlations between 
optimal linear composite. Psychometrika, 49, 11-24.

Steiger, J.H., \& Hakstian, A.R. (1982). The asymptotic distribution of elements of a correlation matrix: Theory and application. British Journal of Mathematical and Statistical Psychology, 35, 208-215.

Steiger, J.H., \& Hakstian, A.R. (1983). A historical note on the asymptotic distribution of correlations. British Journal of Mathematical and Statistical Psychology, 36, 157.

Takeuchi, K., Yanai, H., \& Mukherjee, B.N. (1982). The foundations of multivariate analysis. New Delhi: Wiley Eastern.

Timm, N.H., \& Carlson, J.E. (1976). Part and bipartial canonical correlation analysis. Psychometrika, 41, 159-176.

van den Burg, W., \& Lewis, C. (1988). Some properties of two measures of multivariate association. Psychometrika, 53, 109-122.

van den Burg, W., \& Lewis, C. (1990). Testing multivariate partial, semipartial and bipartial correlation coefficients. Multivariate Behavioral Research, 25, 335-340.

Wilks, S.S. (1932). Certain generalizations in the analysis of variance. Biometrika, 24, 471-494.

(Received April 20 2007, Revised December 18 2007) 